\title{
FUNDAMENTALS-LONGITUDINAL MOTION*
}

\author{
W. T. Weng
}

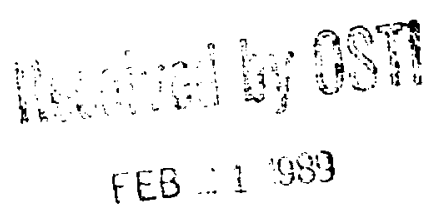

November 7, 1988

Accelerator Development Department

Brookhaven National Laboratory, Upton, NY 11973

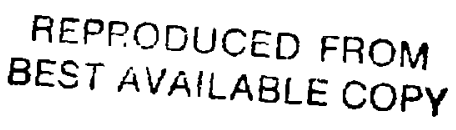

* Lecture given at the "US Summer School on High Energy Particle Accelerators", August, 1987, Fermilab. 


\title{
FUNDAMENTALS-LONGITUDINAL MOTION*
}

\author{
W. T. Weng
}

November 7, 1988

\author{
Accelerator Development Department \\ Brookhaven National Laboratory, Upton, NY 11973
}

\section{DISCLAIMER}

\begin{abstract}
This report was prepared as an account of work sponsored by an agency of the United States Government. Neither the United States Government nor any agency thireof, nor any of their employees, makes any warranty, express or implied, or assumes any legal liability or responsibility for the accuracy, completeness, or usefulness of any information, apparatus, product, or process disclosed, or represents that its use would not infringe privately owned rights. Reference herein to any specific commercial product, process, or service by trade name, trademark, manufacturer, or otherwise does not necessarily constitute or imply its endorsement, recommendation, or favoring by the United States Government or any agency thereof. Tr : views and opinions of authors expressed herein do not necessarily state or reflect those of the United States Government or any agency thereof.
\end{abstract}

" Lecture given at the "US Summer School on High Energy Particle Accelerators", August, 1987, Fermilab. 


\section{FUNDAMENTALS-LONGITUDINAL MOTION}

W. T. Weng

Brookhaven National Laboratory, Upton, NY 11979

TABLE OF CONTENTS

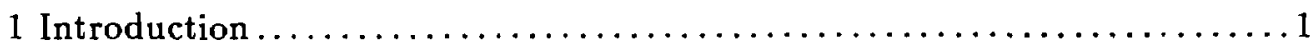

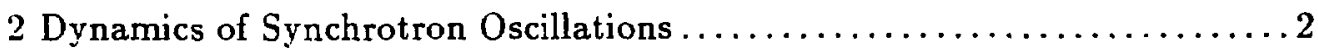

2.1 Equations of Motion .....................................

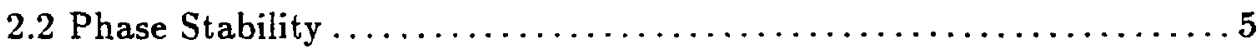

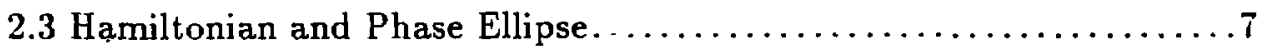

2.4 Oscillation Amplitudes and Limits of Stable Region .............9

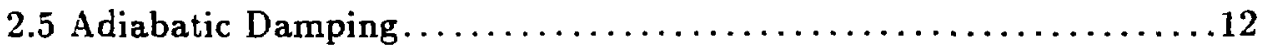

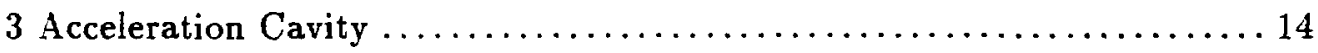

4 Beam Manipulation and Beam Control $\ldots \ldots \ldots \ldots \ldots \ldots \ldots \ldots \ldots \ldots$

4.1 RF Capture, Bunching, and Debunching ................... 19

4.2 Synchronous Transfer and Phase-Space Matching ............23

4.3 Perturbed Synchronous Oscillations.....................24

4.4 Beam Loading and Robinson Instability $\ldots \ldots \ldots \ldots \ldots \ldots \ldots \ldots$

5 High Intensity Effects...............................

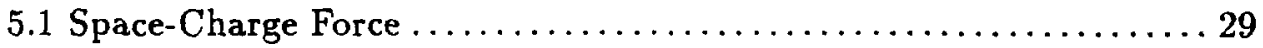

$5.2 \gamma_{t r^{-}}$Jump at Transition $\ldots \ldots \ldots \ldots \ldots \ldots \ldots \ldots \ldots \ldots \ldots \ldots \ldots \ldots \ldots \ldots \ldots \ldots$

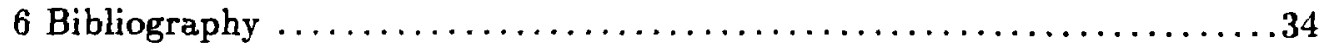




\title{
FUNDAMENTALS-LONGITUDINAL MOTION
}

\author{
W. T. Weng \\ Brookhaven National Laboratory, Upton, NY 11973
}

\section{INTRODUCTION}

There are many ways to accelerate charged particles to high energy for physics research. Each has served its purpose, but eventually has encountered fundamental limitations of one kind or another. Looking at the famous Livingston curve (Fig. 1), we see the initial birth and final level-off of all types of accelerators. In fact, in the mid-80s we personally witnessed the creation of a new type of collider-the Stanford Linear Collider. We also witnessed the resurgence of study into novel methods of acceleration.

You are going to hear a lot of discussion about other methods of acceleration in this two-week period. For my part, I will cover acceleration and longitudinal motion in a synchrotron.

A synchrotron is a circular accelerator with the following three characteristics:

1. Magnetic guiding (dipole) and confinement (quadrupole) components are placed in a small neighborhood around the equilibrium orbit.

2. Particles are kept in resonance with the radio-frequency electric field indefinitely to achieve acceleration to higher energies.

3. Magnetic fields are varied adiabatically with the energy of the particle.

D. Edwards described the transverse oscillations of particles in a synchrotron. I will talk about the longitudinal oscillations of particles. The phase stability principle was invented by V. Veksler and E. McMillan independently in 1945. The Phase stability and strong focusing principle, invented by Courant 


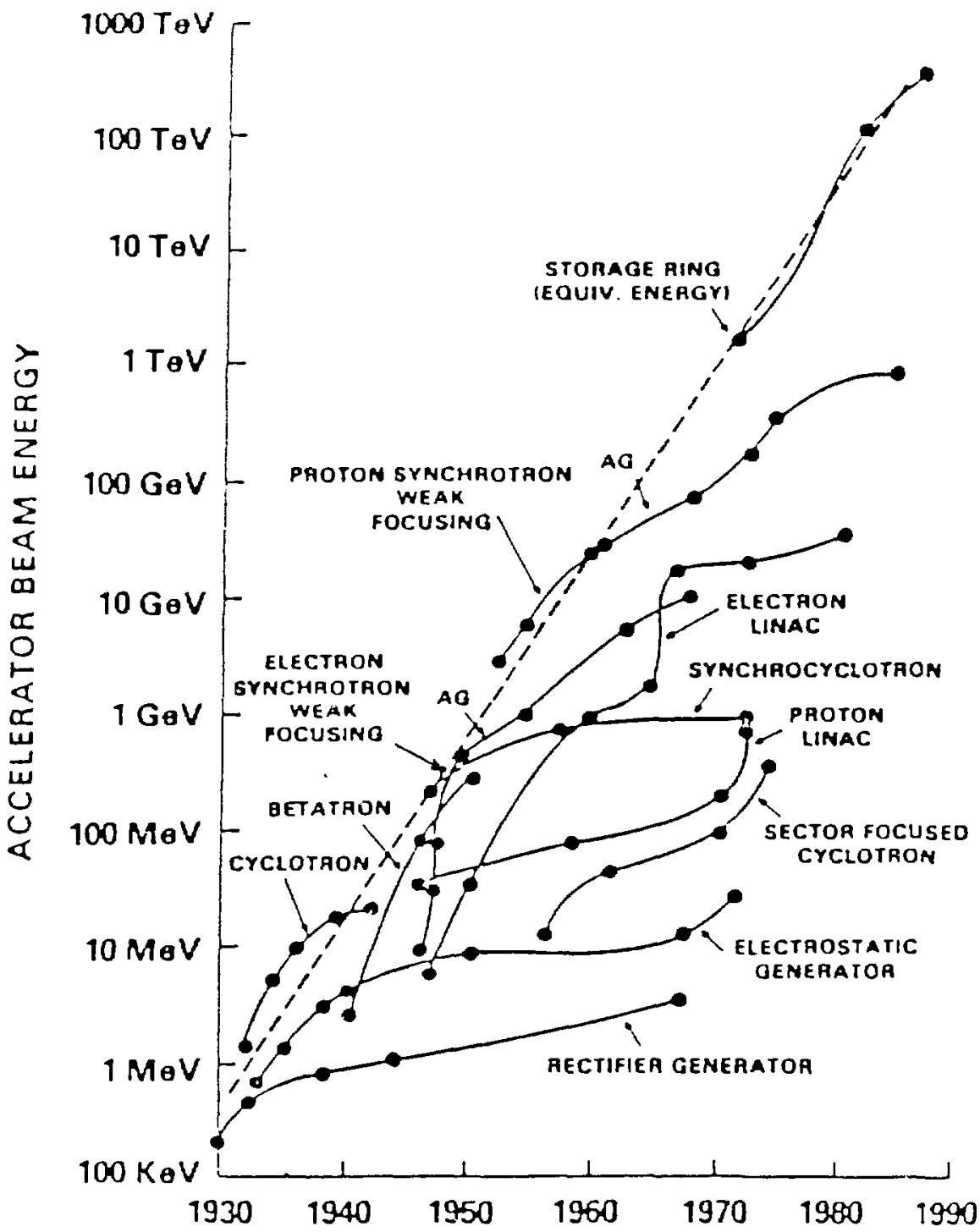

Figure : Energy growth of accelerators and storage -ings. 
and Livingston in 1952, enabled the steady energy gain of accelerators and storage rings witnessed during the past 30 years.

What I will discuss here is a well-established and well-practiced subject. Many review papers and research articles have been written about this rich and interesting field. It is very hard to be original about them. Most of the material here is drawn from references 4,9 , and 15.

What I hope to add is a unified overview of the related rf subjects in an accelerator and a close coupling between accelerator physics and engineering practices, which is essential for the major progress in such areas such as high intensity synchrotrons, multistage accelerator complex, and anti-proton production and cooling, made possible in the past 20 years. I also hope that after this summer school, you will have the basic knowledge to let you understand the discussion and to ease the way for you to dip into the field if you so choose.

This talk overlaps with and leads naturally to other lectures in this school, in particular, those by Humphries, Puglisi, and Gareyte.

\section{DYNAMICS OF SYNCHROTRON OSCILLATIONS}

The major components of an accelerator are shown in Fig. 2. There is a closed (equilibrium) orbit which represents the stationary trajectory if a particle is launched onto it. A particle not exactly on the closed orbit will execute quasi-periodic oscillations around the closed orbit. These are the socalled betatron oscillations and they form the subject of Edwards' lectures.

During acceleration, a radio-frequency voltage is generated across the gap of an accelerating cavity. Again, there is a particle which arrives at the gap at the proper time to receive a predetermined energy gain to stay on the closed orbit that is governed by the guide field. Such a particle is called a synchronous 


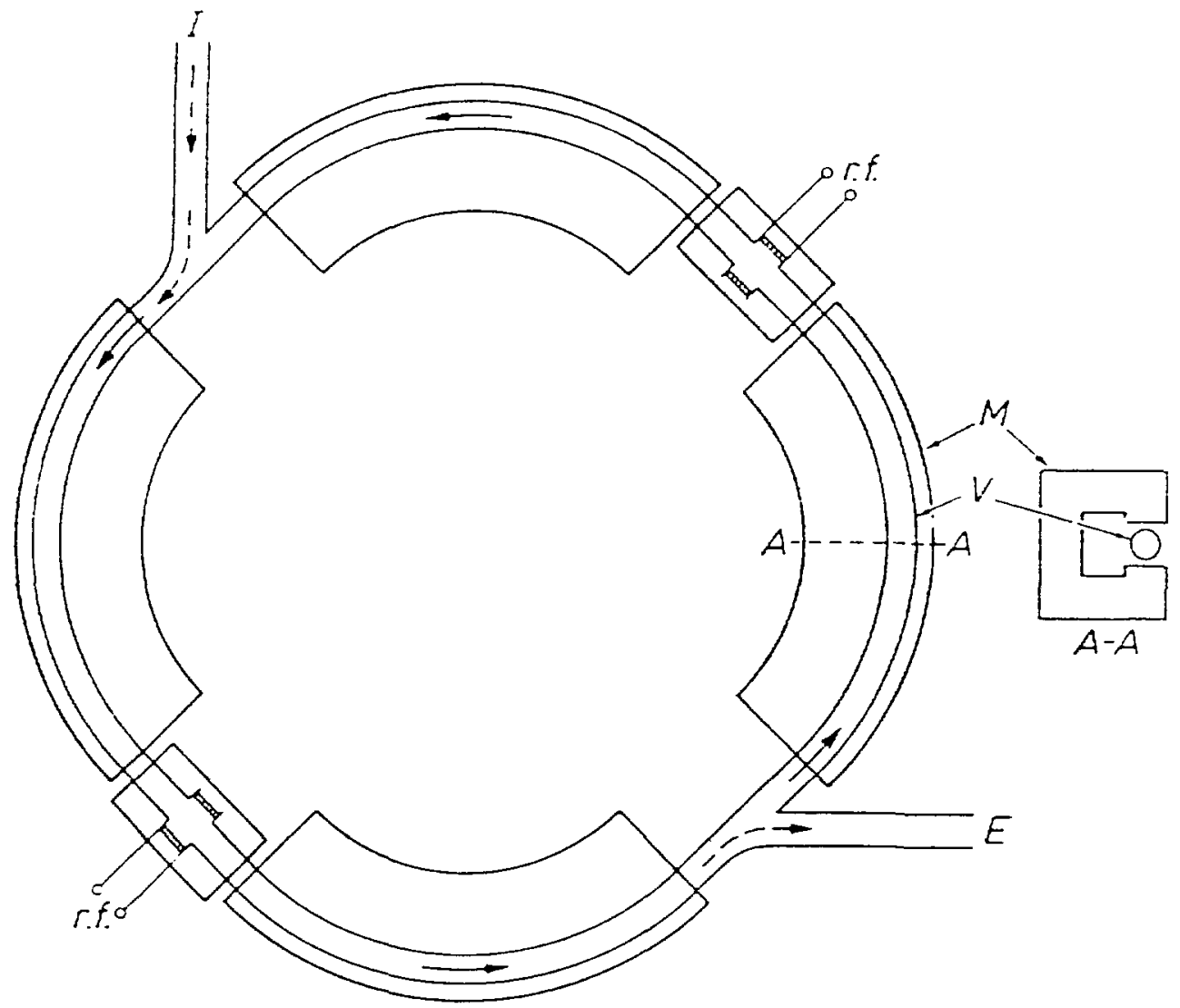

Fig.2. A proton sychrotron, schematic. The protons are kept in a closed orbit in the vacuum chamber $V$ by guiding magnets $\mathrm{H}$. Betveen magnets the orbit is straight. Straight sections provide room for accelerating the protons (r.f.), for injecting them at the besmonity of a cyele ( $I$ ) and for ejecting them at the end $(E)$. 
particle (SP). Other particles in the vicinity of the synchronous particle will execute synchrotron oscillations in both energy and of phase deviations. Because the phase deviation is manifested as a coordinate deviation in the lcagitudinal direction, the synchrotron motion is also referred to as longitudinal motion.

\section{I EQUATIONS OF MOTION}

Mathematically, the voltage across the gap of the accelerating cavity can be expressed as

$$
V(t)=\hat{V} \sin \phi(t)
$$

The voltage wave form is shown in Fig. 3. The rf frequency $\omega$ is an integral multiple $h$ (harmonic number) of the revolution frequency $\Omega_{\circ}$ of the synchronous particle,

$$
\omega=h \Omega_{\circ}=\frac{h \beta_{\circ} C}{R_{\circ}},
$$

where $R_{0}$ is the average radius of the accelerator and $\beta_{0} C$ is the velocity of the particle. The average bending field $B_{\circ}$ on the synchronous orbit of a particle with momentum $P_{\text {。 }}$ is given by

$$
B_{\circ}=\frac{P_{\circ}}{e R_{\circ}}
$$

The energy gain of the SP in one turn is given by

$$
\Delta E_{\circ}=e \hat{V} \sin \phi_{\circ}=2 \pi e R_{\circ}^{2} \dot{B}_{\circ}
$$

Equation (2.4) specifies the required rate of adiabatic change of the guiding field to keep the SP on the design closed orbit. During acceleration a feedback system is often provided to keep the particles' excursions from the design 


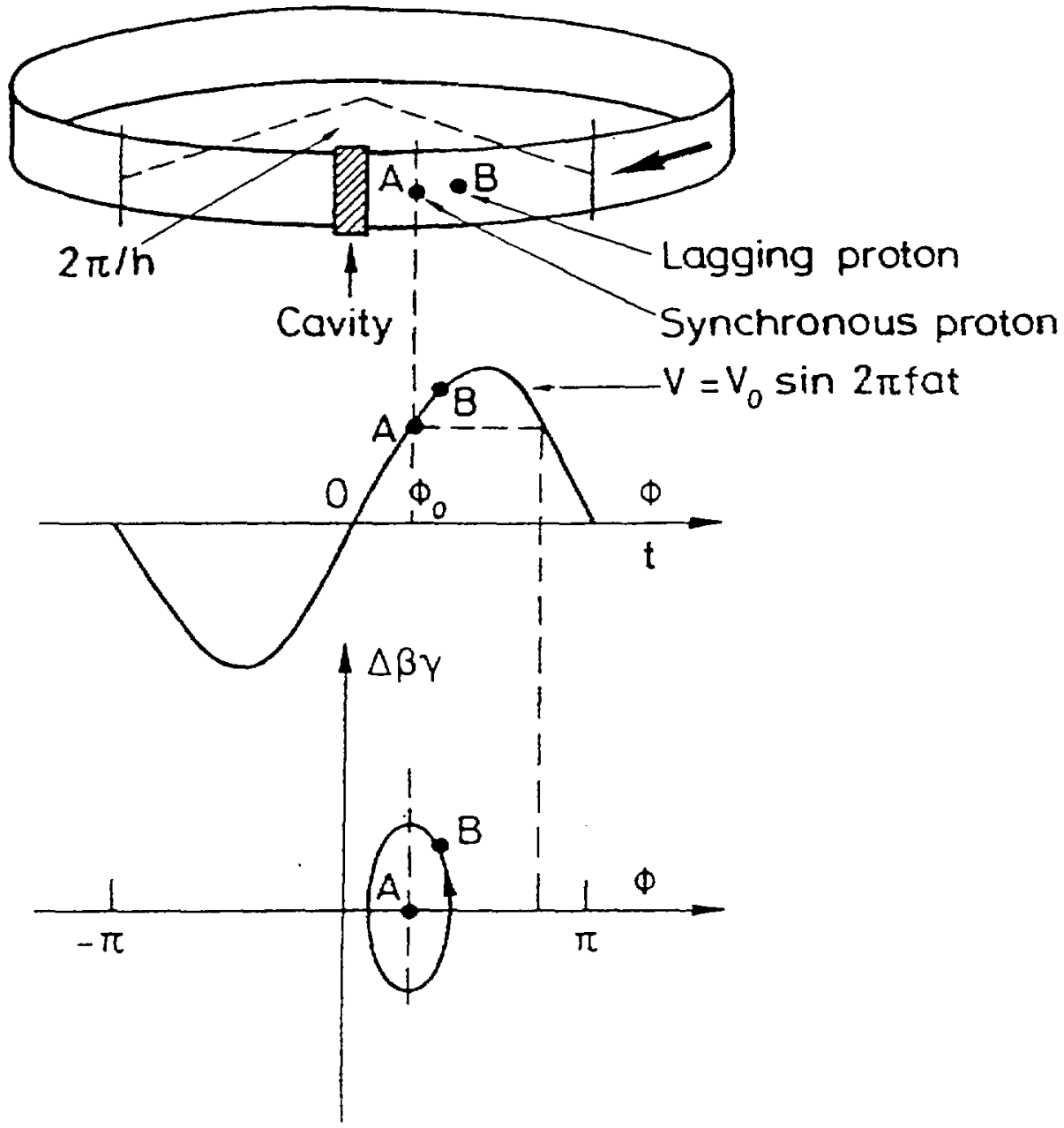

Fig 3. Cylindrical coordinate system rotates with beam, demonstrates meaning of RF phase angle in longitudinal phase space 
orbit to a minimum and another (phase) loop is provided to keep the SP in phase with the rf voltage at the gap. From now on we will concentrate on the equations of motion of the non-synchronous particles (NSP).

The parameters of a NSP not too far from the SP can be expressed as

$r=r_{\circ}+\Delta r, \quad \phi=\phi_{\circ}+\Delta \phi, \quad P=P_{\mathrm{o}}+\Delta P, \quad E=E_{\mathrm{o}}+\Delta E, \quad \theta=\theta_{\mathrm{o}}+\Delta \theta$,

where $\theta$ is the azimuthal angle around the machine. We have

$$
\Delta \phi=-h \Delta \theta, \quad \Delta \Omega=-\frac{1}{h} \frac{d \phi}{d t}
$$

One quantity that is important in the discussion of longitudinal motion is the frequency slip factor $\eta$. It is defined as

$$
\eta \equiv-\frac{d \Omega / \Omega}{d P / P}=\frac{1}{\gamma_{t r}^{2}}-\frac{1}{\gamma^{2}}
$$

where $\gamma_{t r}$ is the transition energy of the synchrotron. From Eq. (2.7), $\eta$ is negative below transition, meaning a higher energy particle has a $h_{k}$ her frequency in the ring; and it is positive above transition, meaning a higher energy particle has a lower frequency. This is due to the balance between increase in speed and increase in path length, due to dispersion function, of the higher energy particle.

The equations of motion of a NSP are

$$
\frac{d}{d t}\left(\frac{\Delta E}{\Omega_{\mathrm{o}}}\right)=\frac{e \hat{V}}{2 \pi}\left[\sin \phi-\sin \phi_{\mathrm{o}}\right]
$$

and 


$$
\frac{d \phi}{d t}=\frac{h \eta \Omega_{\circ}}{P_{\circ} R_{\circ}}\left(\frac{\Delta E}{\Omega_{\circ}}\right) .
$$

These two first-order differential equations can be combined into one secondorder differential equation,

$$
\frac{d}{d t}\left[\frac{P_{\circ} R_{\circ}}{h \eta \Omega_{\circ}} \frac{d \phi}{d t}\right]-\frac{e \hat{V}}{2 \pi}\left[\sin \phi-\sin \phi_{\circ}\right]=0 .
$$

Equation (2.10) is exact for all the acceleration cycle. Often, however, the acceleration is an adiabatic process. It is then useful to neglect the small change in momentum and work with small phase deviations. We then get the simplified linearized equation of small-amplitude synchrotron oscillations,

$$
\frac{d^{2}}{d t^{2}}(\Delta \phi)-\frac{e \hat{V} \eta \Omega_{\circ} \cos \phi_{\circ}}{2 \pi P_{\circ} R_{\circ}}(\Delta \phi)=0
$$

\subsection{PHASE STABILITY}

Equation (2.11) has stable oscillatory solutions if the constant coefficient of the equation is positive, or

$$
\eta \cos \phi_{\circ}<0
$$

which is realizable in a synchrotron in two situations for acceleration:

a) below transition

$$
\gamma<\gamma_{t r}, \quad \eta<0,0<\phi_{\circ}<\frac{\pi}{2}, \sin \phi_{\circ}>0
$$

b) above transition

$$
\gamma>\gamma_{t r}, \eta>0, \frac{\pi}{2}<\phi_{0}<\pi, \sin \phi_{0}>0
$$


The relation between the if voltage and the phase ellipse, to be explained shortly, is shown in Fig. 4. Two other solutions for deceleration exist which can be worked out by the readers.

Let us look at the mechanism of "phase stability" in more detail by following the motion of a NSP below transition. A lagging particle B will gain energy at the crossing of the if cavity and this will cause it to speed up and overtake the SP. At a certain point, its energy gain will make it arrive at the gap earlier than the SP and particle B will therefore obtain less energy from the If station than the SP. Then, the reverse process starts. This motion up and down the linear part of the rf wave is the basic mechanism of phase stability and is reminiscent of simple harmonic motion.

Because the coefficient of the differential equation is positive, the frequency of the small-amplitude synchrotron oscillations $\Omega$, is given by

$$
\frac{d^{2}}{d t^{2}}(\Delta \phi)+\Omega_{\cdot}^{2}(\Delta \phi)=0
$$

where

$$
\Omega_{\bullet}=\left[-\frac{e \hat{V} \eta \Omega_{0} \cos \phi_{0}}{2 \pi P_{0} R_{\circ}}\right]^{1 / 2}
$$

The ratio of the synchrotron oscillation frequency $\Omega$, to the revolution frequency $\Omega_{0}$ is often called the tune of the synchrotron oscillations and denoted by $\nu_{0}$,

$$
\nu_{\star} \equiv \frac{\Omega_{0}}{\Omega_{0}}
$$

which generally lies in the range 0.001 to 0.01 for proton synchrotrons and is usually much smaller than the tune of the betatron oscillations. In an electron 

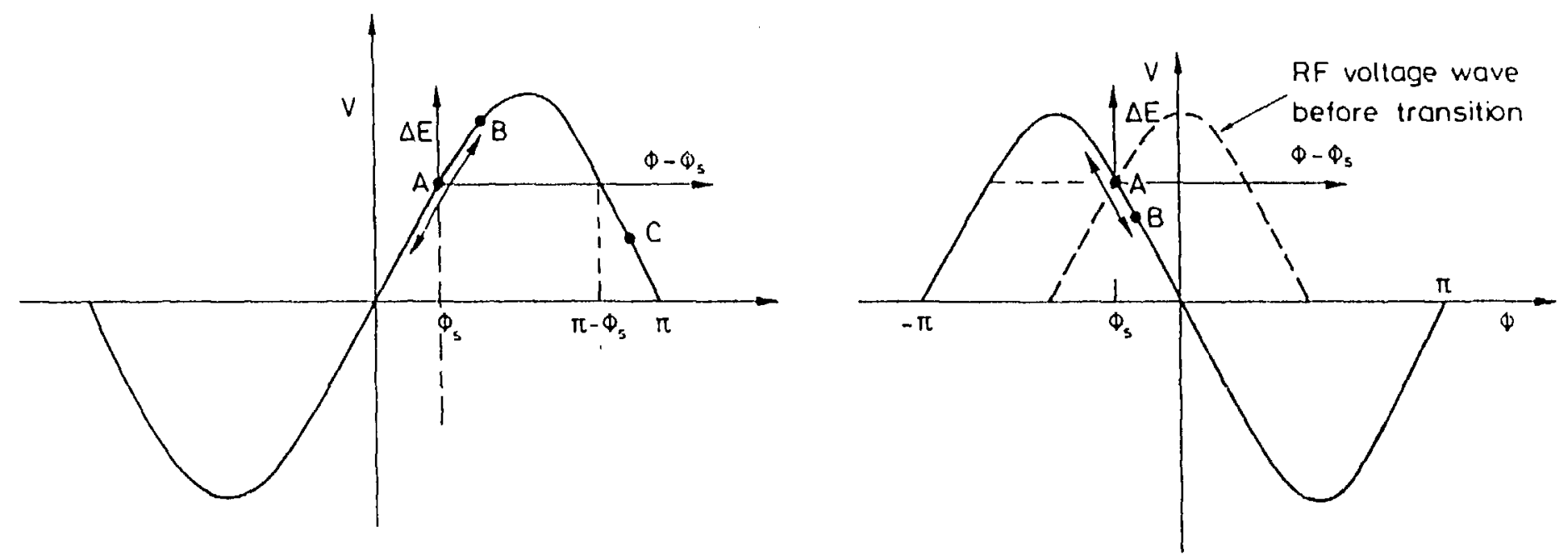

a

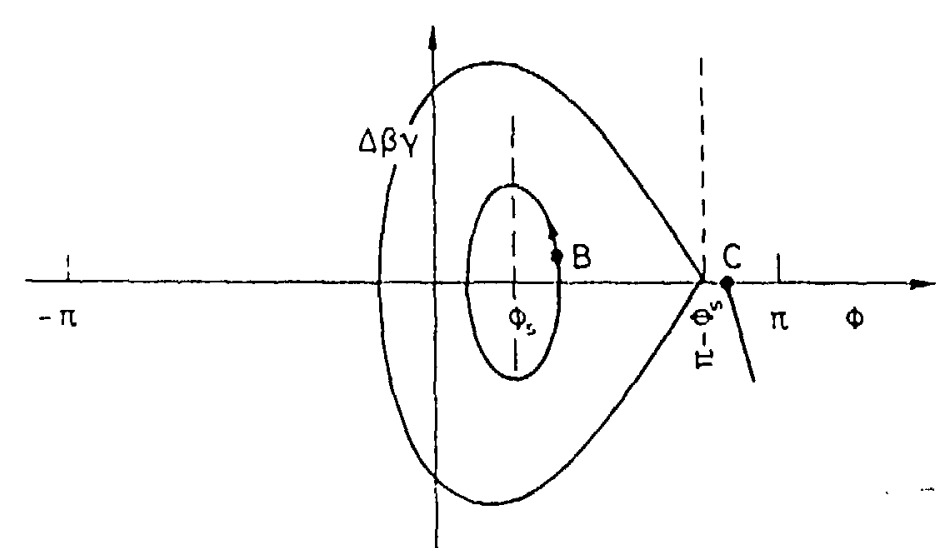

a)

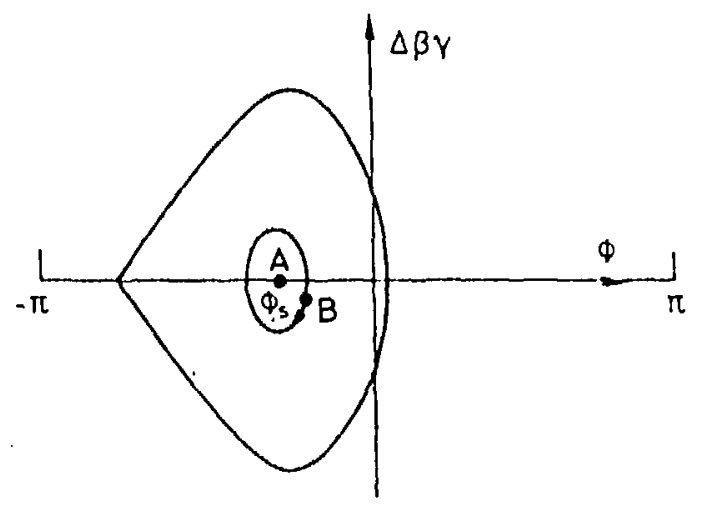

b)

Fig. 4. Plase stability of synchrotron motion below (a) and above (b) transition 
synchrotron, $\nu$, can be as high as 0.2 , where the coupling of betatron and synchrotron oscillations will become more important.

As $\eta$ approaches zero transition energy, sc does $\Omega_{s}$, and there is no phase stability. During acceleration through transition energy in a proton synchrotron, the rf phase must be switched abruptly from $\phi_{\circ}$ to $\pi-\phi_{\circ}$ in order to maintain phase stability above transition. In a real beam with a finite energy spread, the timing of the phase switching cannot be accurate for all the particles, and some blow-up in phase-space area is unavoidable. This effect is further enhanced in the presence of space-charge forces. Various methods, such as multiple phase switching and $\gamma_{t r}$ jump schemes, have been used to minimize the ill effects.

\subsection{HAMILTONIAN AND PHASE ELLIPSE}

All the detailed behavior of particles undergoing synchrotron oscillation can be analysed by working with Eqs. (2.8) and (2.9). But, for a qualitative understanding of bunches of particles, it is often more transparent to work with the Hamiltonian formulation of the same problem. In order to do this, we first have to introduce the "energy" variable $W$ canonically conjugate to the "position" variable $\phi$,

$$
W \equiv \frac{\Delta E}{\Omega_{0}}
$$

which has the dimensions of action (energy times time). The equations of motion (2.8) and (2.9) can be derived from the Hamiltonian

$$
H(\phi, W)=\frac{h \eta \Omega_{\circ}}{2 P_{\circ} R_{\circ}} W^{2}+\frac{e \hat{V}}{2 \pi}\left[\cos \phi-\cos \phi_{\circ}+\left(\phi-\phi_{\circ}\right) \sin \phi_{\circ}\right]
$$


To show that Eq. (2.17) is the correct Hamiltonian, let us recall Hamilton's equations of motion,

$$
\begin{gathered}
\dot{W}=\frac{d}{d t}\left(\frac{\Delta E}{\Omega_{\circ}}\right)=-\frac{\partial H}{\partial \phi}=\frac{e \hat{V}}{2 \pi}\left[\sin \phi-\sin \phi_{\circ}\right], \\
\dot{\phi}=\frac{d \phi}{d t}=\frac{\partial H}{\partial W}=\frac{h \eta \Omega_{\circ}}{P_{\circ} R_{\circ}} W,
\end{gathered}
$$

which are identical to Eqs. (2.8) and (2.9). The parameters $\eta, \Omega_{\circ}, \phi_{\circ}, P_{\circ}$, and $\hat{V}$ can vary with time, and the Hamiltonian is therefore time dependent. In most cases, however, the variation is slow enough to be neglected for the discussion of synchrotron motion over a few periods of oscillations, in which case

$$
\frac{d H}{d t}=\frac{\partial H}{\partial t}=0
$$

For this to be true, the fractional change in synchrotron frequency during one radian of oscillation must be small, a condition expressed by the dimensionless adiabaticity parameter

$$
\epsilon=\frac{1}{\Omega_{g}^{2}} \frac{d \Omega_{g}}{d t} \ll 1 .
$$

The motion of the particles is conveniently represented by their trajectories in the $(\phi, W)$ phase plane. These trajectories are contours of constant Hamiltonian (given by Eq. 2.17). Families of such curves are shown in Fig. 5 for $\gamma>\gamma_{t r}$ for three values of the synchronous phase angle $\phi_{0}$.

Particles move in time along the trajectories, in the direction of the arrows, according to Eqs. (2.18) and (2.19). In each interval of $2 \pi$ are two fixed points where $\dot{\phi}=\dot{W}=0$. The stable fixed point (SFP) at $\phi=\phi_{0}, W=0$, 

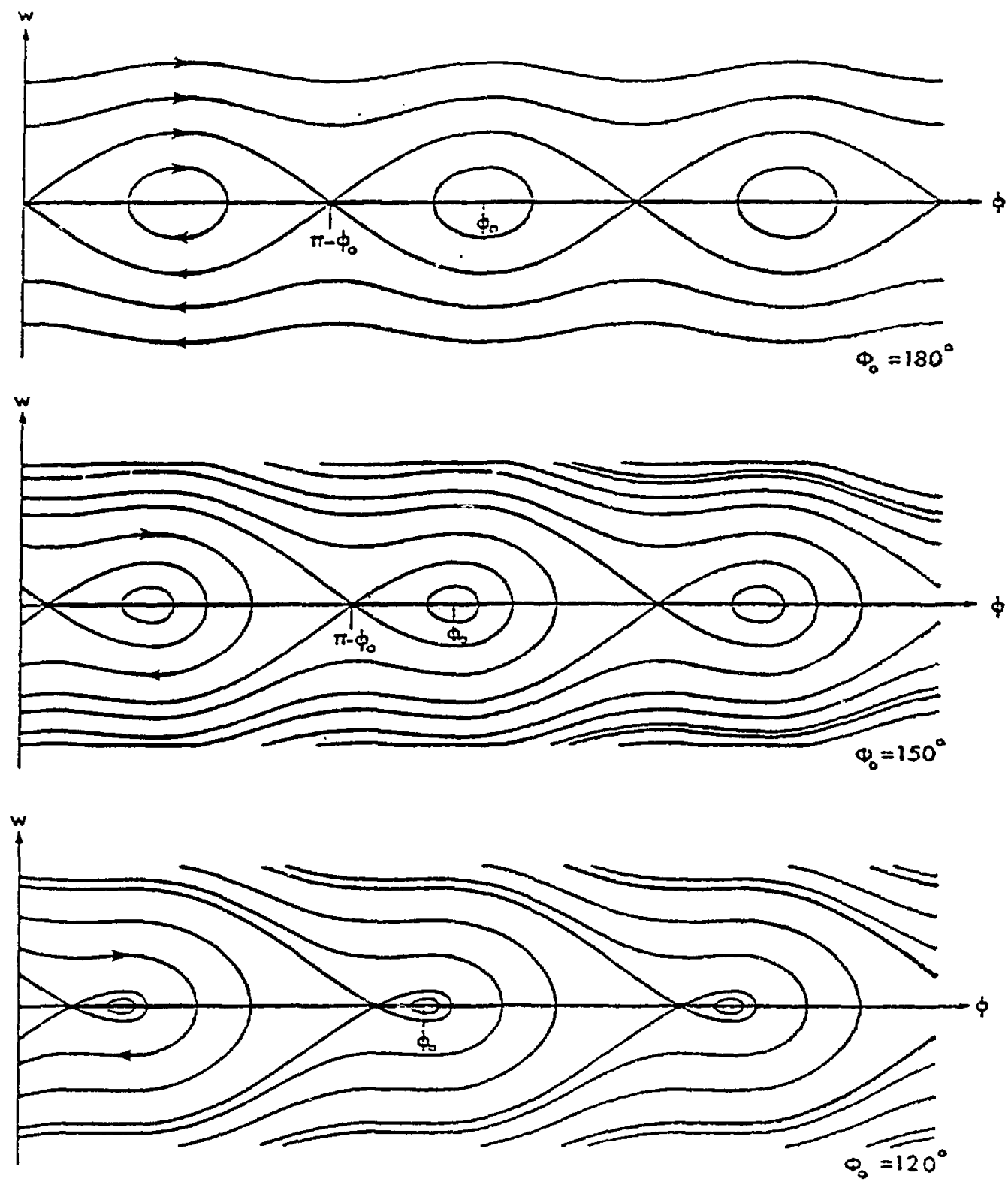

Fig. 5 Trajectories of constart tamiltonian, $\gamma>Y_{L}$ 
corresponding to the synchronous particle, lies inside the stable region (or bucket) bounded by the separatrix. The unstable fixed point (UFP) is at $\phi_{u}=\pi-\phi_{\circ}, W=0$ at the extreme limit of the separatrix. The motion of the particles becomes infinitely slow as they approach this point.

The case of $\phi_{0}=\pi$ corresponds to zero acceleration, $\dot{P}_{0}=0$, or "stationary bucket." The separatrix extends between adjacent UFPs, and trajectories outside the stable region do not cross the line dividing the upper and lower half-planes. The situation is illustrated in Fig. 6.

At small amplitudes, the Hamiltonian can be further reduced to

$$
H=\frac{h \eta \Omega_{\circ}}{2 P_{\circ} R_{\circ}} W^{2}-\frac{e \hat{V}}{2 \pi} \cos \phi_{\circ} \frac{(\Delta \phi)^{2}}{2} .
$$

The constant Hamiltonian curve is hence an ellipse in $(\Delta \phi, W)$ phase space.

\subsection{OSCILLATION AMPLITUDES AND LIMITS OF STABLE REGION}

For a particle inside the stable region, the limits of oscillation in $W$ are given by $\dot{W}=0$, for which Eq. (2.18) gives two solutions,

$$
\phi=\phi_{0},
$$

and

$$
\phi=\pi-\phi_{\circ} \equiv \phi_{u},
$$

this second solution corresponding to the UFP. The first solution substituted into Eq. (2.17) gives the value of the Hamiltonian for the trajectory having extremum $\hat{W}$ in amplitude, and we can write 


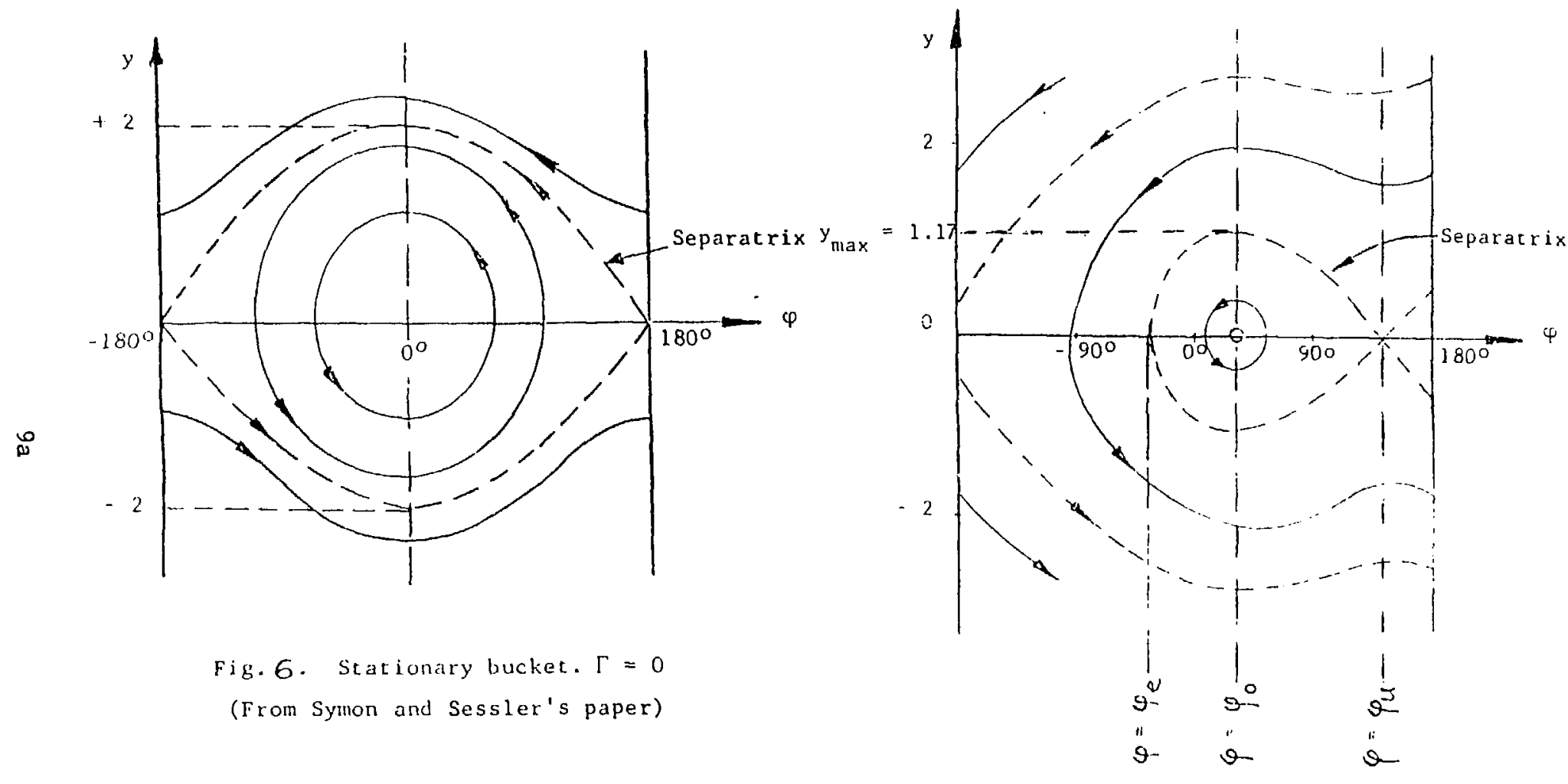

F1g, 7, Moving bucket, Case of $\Gamma=1 / 2$ 


$$
H(\phi, W)=\frac{h \eta \Omega_{\circ}}{2 P_{\mathrm{o}} R_{\mathrm{o}}} \hat{W}^{2}=\frac{h \eta \Omega_{\circ}}{2 P_{\mathrm{o}} R_{\mathrm{o}}} W^{2}+\frac{e \hat{V}}{2 \pi}\left[\cos \phi-\cos \phi_{\circ}+\left(\phi-\phi_{\circ}\right) \sin \phi_{\circ}\right] .
$$

From Eq. (2.19), the extrema in phase are given by $W=0$. Let us look at two interesting special cases.

Case 1: For stationary buckets $\left(\eta<0, \phi_{0}=0\right)$, putting $W=0$ into Eq. (2.25) yields

$$
\frac{h \eta \Omega_{\circ}}{2 P_{\mathrm{o}} R_{\mathrm{o}}} \hat{W}^{2}=\frac{e \hat{V}}{2 \pi}[\cos \phi-1]=-\frac{e \hat{V}}{\pi} \sin ^{2}\left(\frac{\phi}{2}\right),
$$

which in turn gives the limits of oscillation amplitudes in phase

$$
\phi_{\ell}= \pm 2 \sin ^{-1}\left[\frac{\pi h(-\eta) \Omega_{0}}{2 e \hat{V} P_{\mathrm{o}} R_{\mathrm{o}}} \hat{W}^{2}\right]^{1 / 2}
$$

Case 2: For a non-stationary bucket in the small-amplitude approximation and putting

$$
\Delta \phi=\phi-\phi_{\circ} \ll 1,
$$

Eq. $(2.25)$ at $W=0$ yields

$$
\Delta \phi= \pm\left[\frac{2 \pi h(-\eta) \Omega_{\circ}}{2 e \hat{V} P_{\mathrm{o}} R_{\mathrm{o}} \cos \phi_{\mathrm{o}}}\right]^{1 / 2} W
$$

The ratio $W / \Delta \phi$ is an important parameter in matching bunches of particles into RF buckets, for example when transferring a be $ı m$ from one accelerator to another.

We have already seen that the separatrix limiting the phase stable region terminates at the UFP $W=0, \phi_{u}=\pi-\phi_{0}$. We can, therefore, substitute 
these values into Eq. (2.25) in order to determine the extreme amplitudes in $W$ of the separatrix. In other words, these extrema $(W)_{\text {sep }}$ are given by

$$
\frac{\pi h \eta \Omega_{\circ}}{2 e P_{\mathrm{o}} R_{\mathrm{o}}}(W)_{\text {sep }}^{2}=\frac{e \hat{V}}{2 \pi}\left[-\cos \phi_{\circ}+\left(\pi-2 \phi_{\circ}\right) \sin \phi_{\circ}\right]
$$

The other extremum of phase $\phi_{e}$, is obtained by using Eq. (2.29) in Eq. (2.25) with $W=0$. Then,

$$
\cos \phi_{e}-\phi_{e} \sin \phi_{0}=\left(\pi-\phi_{0}\right) \sin \phi_{\circ}-\cos \phi_{0}
$$

This transcendental equation in $\phi_{0}$ reduces to $\cos \phi_{0}=-1,+1$ for $\phi_{0}=0, \pi$ and thus yields the adjacent UFP, as expected for stationary buckets. (See Figs. 6 and 7.)

Another useful quantity to be derived from Eq. (2.25) is the concept of emittance, or phase-space area of a bunch of particles. In the small-amplitude approximation, Eq. (2.25) reduces to

$$
H=\frac{h \eta \Omega_{\circ}}{2 P_{\circ} R_{\circ}} W^{2}+\frac{e \hat{V}}{2 \pi} \cos \phi_{\circ} \frac{(\Delta \phi)^{2}}{2}=\frac{e \hat{V}}{2 \pi} \cos \phi_{\circ} \frac{(\Delta \hat{\phi})^{2}}{2} .
$$

In terms of the bunch length $(\Delta \hat{\phi})$, Eq. (2.31) becomes

$$
\frac{(\Delta \phi)^{2}}{(\Delta \hat{\phi})^{2}}+\frac{h \eta \Omega_{\circ}}{2 P_{\circ} R_{\circ}} \frac{4 \pi}{e \hat{V} \cos \phi_{\circ}(\Delta \hat{\phi})^{2}} W^{2}=1
$$

which is the standard equation of an ellipse, and the area of the phase-space ellipse is given by

$$
A_{\mathrm{g}}=\pi a b=\pi(\Delta \hat{\phi})^{2}\left[\frac{e \hat{V} \cos \phi_{\mathrm{o}} P_{\mathrm{o}} R_{\mathrm{o}}}{2 h \eta \Omega_{\mathrm{o}} \pi}\right]^{1 / 2} .
$$

This parameter, the longitudinal emittance of the beam, stays constant during the acceleration cycle and is an important parameter governing beam 
quality in a synchrotron or storage ring. In terms of $\hat{W}$ and $\Delta \hat{\phi}$, the area becomes $A_{0}=\pi \hat{W} \Delta \hat{\phi}$. Since $A_{0}$ is an invariant quantity during acceleration, to be proved in the next section, it is often convenient to express $\hat{W}$ or $\Delta \hat{\phi}$ in terms of $A_{\text {. }}$.

\subsection{ADIABATIC DAMPING}

We have so far assumed a constant Hamiltonian which satisfies the adiabaticity condition (2.21). In a synchrotron, however, the particles do receive acceleration, and, thercfore we must study the long-term evolution of the synchrotron motion under adiabatic changes of parameters. Since $(\phi, W)$ is a pair of canonically conjugate variables, Liouville's theorem holds, and therefore arbitrary areas in the $(\phi, W)$ phase plane are conserved in a canonical transformation. With changing parameters however, the stable trajectories in the phase plane do not exactly close over one cycle of synchrotron oscillations and it is not then obvious that area conservation is true.

A more general principle is the adiabatic theorem, which states that, if $q$ and $p$ are canonically conjugate variables of an oscillator with slowly changing parameters, then the action integral

$$
I=\oint p \cdot d q
$$

is an invariant. The integral is taken over one period of oscillations. Applying this to the small-amplitude synchrotron oscillations we have

$$
I=\oint W d \phi=\overline{\left(W \frac{d \phi}{d t}\right)} \frac{2 \pi}{\Omega_{s}},
$$

which leads to the time dependence of $\hat{W}$ and $\Delta \hat{\phi}$ under adiabatic changes of parameters as 


$$
\hat{W} \propto\left(\frac{-P_{\mathrm{o}} R_{\mathrm{o}} \hat{V} \cos \phi_{\mathrm{o}}}{\eta \Omega_{\mathrm{o}}}\right)^{1 / 4}
$$

and

$$
\Delta \hat{\phi} \propto\left(\frac{-\eta \Omega_{0}}{P_{\mathrm{o}} R_{\mathrm{o}} \hat{V} \cos \phi_{\mathrm{o}}}\right)^{1 / 4} .
$$

From Eq. (2.33) we see that, during acceleration, $P_{0}$ increases and the other parameters remain more or less constant, and the phase excursion $\Delta \hat{\phi}$ is reduced as the one-fourth power of the momentum. This is loosely called "adiabatic damping" of phase oscillations. However, the product $\hat{W} \Delta \hat{\phi}$ is an invariant, Liouville's theorem holds, and there is no damping of the area in the phase plane. Figure 8 shows the behavior of $\Delta E$ and $\Delta \phi$ through a typical acceleration cycle, including that near transition energy.

So far we have treated the synchrotron oscillations by setting up a differential equation which implicitly implies a continuous process. This is justifiable because the synchrotron oscillation frequency is much smaller than the particle revolution frequency. However, since the acceleration process in an accelerator is intrinsically a discrete process, a difference equation is natural for describing such an event. Then the energy and phase increments from one cycle to the next are

$$
\begin{gathered}
E_{n}-E_{n-1}=e \hat{V} \sin \phi_{n}, \\
\phi_{n}-\phi_{n-1}=2 \pi h \frac{\omega_{n}\left(E_{n}\right)}{\omega_{n-1}\left(E_{n-1}\right)} .
\end{gathered}
$$

We can solve the synchrotron oscillation problem by solving the difference equations (2.37) and (2.38). Lately, people who worry about the long-term 


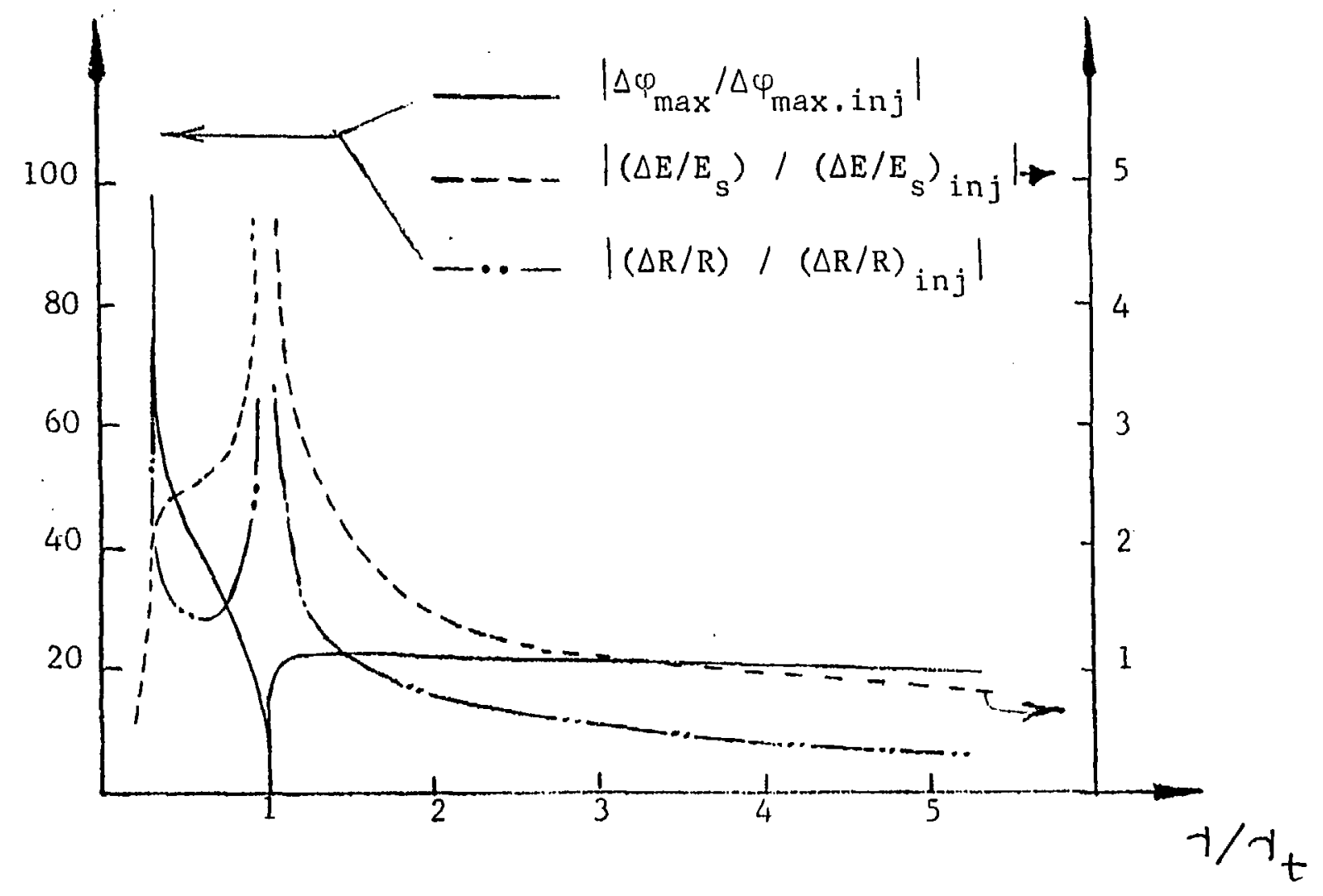

F1g. 8. Bunch phase, energy, and radius as functions of energy。 
stability of synchrotron oscillations in a storage ring, where the number of revolutions can easily exceed the life of the solar system, use Eqs. (2.37) and (2.38) as a mapping from one point in the phase plane to another and use the techniques developed for dynamical systems to ascertain the stability of the oscillations.

Perfectionists consider differential or difference equations not good enough. They set up a computer tracking program to include everything conceivable in a big package.

For what we have talked about, a tracking program is overkill. But, by the time we include multiparticle effects, beam-cavity interactions, and synchrobetatron coupling, we are in the realm of a multiparticle-multidimensional time-dependent problem which no human mind can comprehend in its entirety, and we have to defer to the all-powerful computer. One example of a tracking package for longitudinal motion is ESMP' ${ }^{12}$, created at FNAL, mainly by J. Maclachlan and collaborators, which we will use to demonstrate the capture process in a later chapter.

\section{ACCELERATION CAVITY}

To provide acceleration fields to particles in an accelerator, accelerating stations are required. In general, each accelerating station comprises

(a) a resonator with an accelerating gap,

(b) a power amplifier, and

(c) a frequency tuning device.

Here, we will describe the resonator part of the system to show, in a simple way, how the electrical field is produced and how its performance affects the beam. A more complete description will be given by Puglisi. Computer 
codes used to calculate field distributions and relevant design parameters of an accelerating structure will be covered by Cooper and Jones.

For electron synchrotrons, where the required resonance frequency is in the hundred- $\mathrm{MHz}$ region and there is no need for frequency variation, a hollow pill-box cavity is often used, as shown in Fig. 9a. But for a low energy proton synchrotron, the resonance frequency is typically in the range of a few $\mathrm{MHz}$ to to a few tens of $\mathrm{MHz}$. If an unloaded resonator is used, the physical dimensions will be too large and furthermore the required frequency change to follow the particles' energy increase will be hard to implement. To achieve both goals, a resonator loaded with ferromagnetic materials is often adopted.

The length of the cavity follows the law

$$
\ell \propto \frac{1}{\omega \sqrt{\mu \epsilon}}
$$

where $\mu$ and $\epsilon$ are respectively the permeability and the dielectric constant of the material loading the cavity. Since the space available between the magnet units is restricted, it is advisable to use a material with a rather high permeability (ferrite). For example, if the permeability of the ferrite is $\mu=100$, the length of the resonator is reduced by a factor of 10 . To control the resonance frequency, a dc bias current is supplied to adjust the permeability of the ferrite. To illustrate, schematic diagrams of the AGS rf cavity are shown in Figs. 10 and 11.

Such an accelerating unit has a close resemblance to a resonant cavity consisting of two $\lambda / 4$ coaxial resonators connected face-to-face and operating in the push-pull mode. The cavity resonant frequency is also controlled by an external capacitor. The two halves of the accelerating unit may be referred to as foreshortened $\lambda / 4$ coaxial resonators. One additional advantage of the cavity 


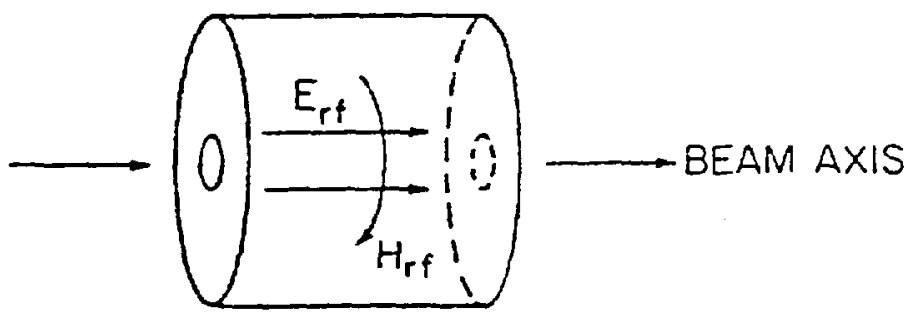

(a)

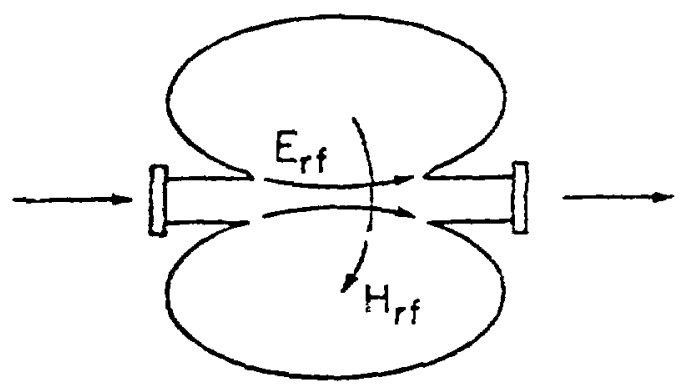

(b)

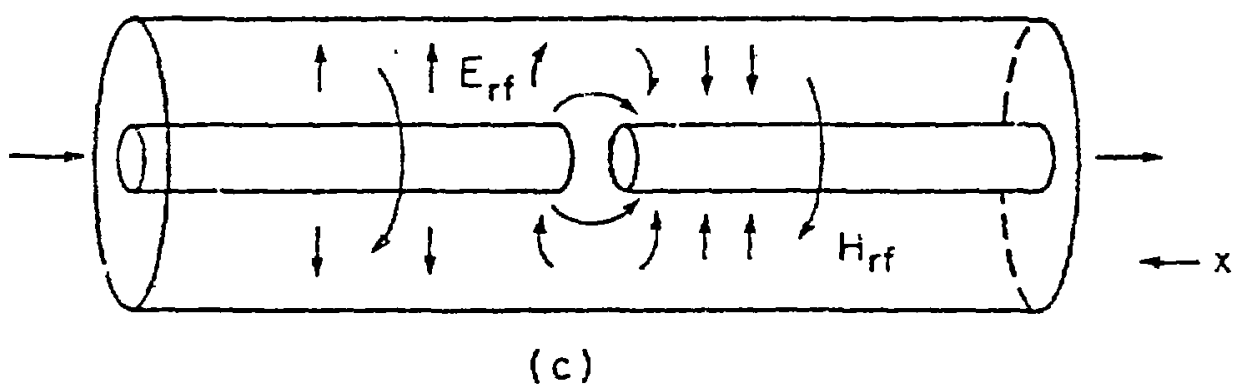

Fig. 9 (a) Simple pill box resonator $T M$ olo mode.

(b) Spherical resonator with nose-cones.

(c) Lower frequency resonator, TEl mode. Suitable for MHz operation. 


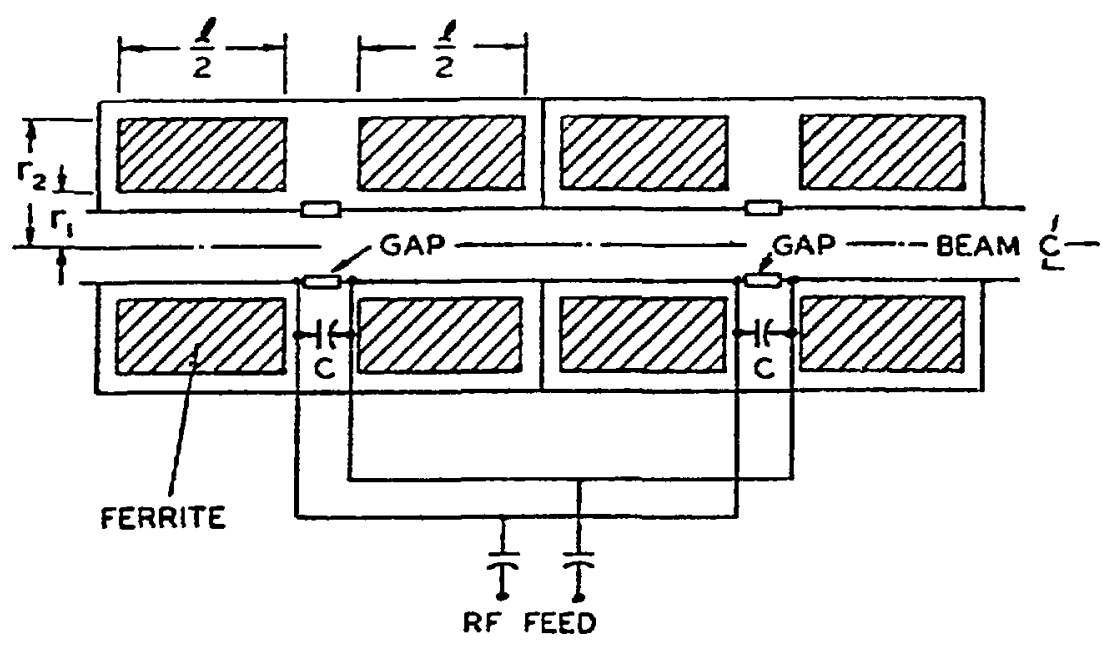

DC BIASING NOT SHOWN

Fig.10. AGS ferrite cavity-schematic representation.

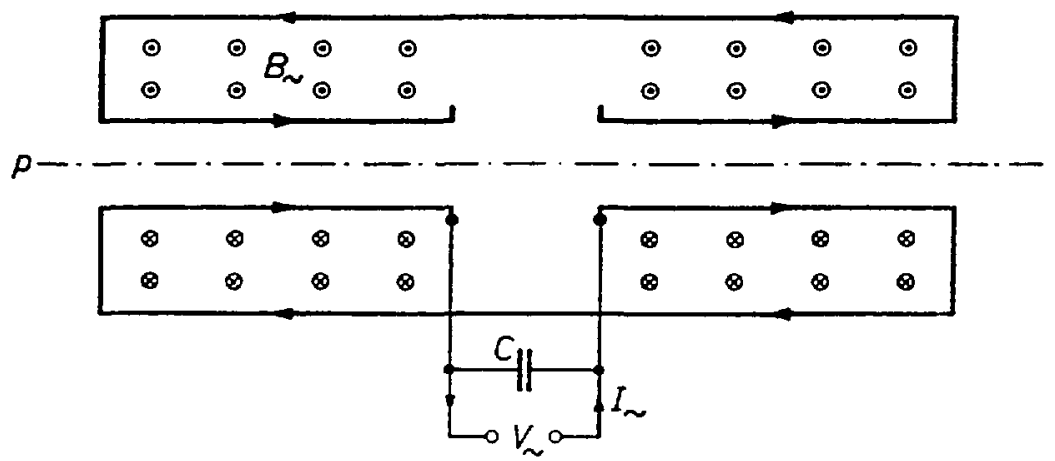

Fig. Il. An accelerating cavity (schematic). The protons move along the axis of the cylinurical structure in a beam $p$. The cavity can be considered as a single-turn inductor. An r.f. current $I$ fed into the cavity wall produces an r.f. magnetic field in the cavity around the axis, and the corresponding r.f. Hux (of density $B_{\sim}$ ) induces an r.f. voltage $V_{\sim}$ across the gap. An external capacitor $C$ may be used to obtain an $L C$ circuit resonant to the operating frequency. 
loaded with ferrite is the large value of the shunt impedance resulting from the increased inductance $L$, which makes the lower exciting current possible.

Assuming that the inner and outer radii of the ferrite rings are $r_{1}$ and $r_{2}$, the inductance of the structure is

$$
L=\frac{\mu \ell}{2 \pi} \ln \left(\frac{r_{2}}{r_{1}}\right)
$$

The capacitance is

$$
C=\frac{2 \pi \epsilon_{o} \ell}{\ln \left(r_{2} / r_{1}\right)}
$$

Neglecting the attenuation due to the small line resistance, we have a real characteristic impedance of the line

$$
Z_{c}=R_{c}=\left(\frac{L}{C}\right)^{1 / 2}=\frac{1}{2 \pi}\left(\frac{\mu}{\epsilon}\right)^{1 / 2} \ln \left(\frac{r_{2}}{r_{1}}\right) .
$$

At resonance, the quality factor $Q$ is

$$
Q=\frac{\omega L}{R}
$$

and the shunt impedance, for a zero gap capacitor, is

$$
R_{a h}=\frac{V^{2}}{P}=\frac{4}{\pi} R_{c} Q
$$

where $R_{s h}$ is the resistance presented by the structure to the beam current in the accelerator.

From the expression for the resonance frequency of the system, $\omega=1 / \sqrt{L C}$, it follows that the bias current has to be capable of reducing the permeability of the ferrite by a factor of 


$$
\frac{\mu_{\max }}{\mu_{\min }}=\left(\frac{f_{f}}{f_{i}}\right)^{2},
$$

where $f_{i}$ and $f_{f}$ are the initial and final frequency respectively. For example, for the AGS, $f_{i}=2.4 \mathrm{MHz}$ and $f_{f}=4.45 \mathrm{MHz}$, implying that the permeability tuning range is 3.4. This is the frequency range for accelerating protons. Last year the AGS started to accelerate heavy ions, which have a much lower injection energy, hence oscillation frequency. The original AGS rf cavity was incapable of tuning to accommodate such a wide range of frequency swing. Therefore, a second set of $\mathrm{rf}$ stations had to be built to preaccelerate the heavy ions to higher energy and then switch to the original if station for further acceleration.

Now let us look at the relation between the voltage at the gap and the magnetic field in the ferrite. If the cavity is short and the ferrite permeability $\mu \gg 1$, the voltage is given by

$$
V=\frac{\partial \phi}{\partial t}=\omega \int_{r_{1}}^{r_{2}} B(r) \ell d r .
$$

If $\mu$ is constant at constant radius, then the flux density varies as $r^{-1}$ in a coaxial structure. In the absence of dc bias,

$$
B(r)=B\left(r_{1}\right) \frac{r_{1}}{r}=B_{1} \frac{r_{1}}{r},
$$

and

$$
V=\omega \ell B_{1} r_{1} \ln \left(\frac{r_{2}}{r_{1}}\right) .
$$

For a given structure and power amplifier, one way to get higher voltage at the gap is to increase the outer radius of the ferrite. Any cavity-like discontinuity 
in the vacuum environment represents a source of impedance to the particle beam. The frequency of the impedance is determined by the length of the structure, and the amplitude is related to the depth, as shown in Eq. (3.10).

At the frequency applicable to low energy proton synchrotrons, a lumped RLC circuit is sufficient to represent the accelerating cavity. The equivalent circuit driven by both generator and beam current is shown in Fig. 12. We will refer to this equivalent circuit later in the discussion of beam-loading effects.

For a given cavity design, there are other, higher-order, resonant modes. A spectral analysis of the impedance shows that the $x$ cavity seen by the beam consists mainly of sharp peaks at frequencies corresponding to the cavity modes. Besides being driven at the fundamental longitudinal accelerating mode, the cavities will also be driven by the beam at their higher-order longitudinal and transverse parasitic-modes which lie at higher frequencies (up to the cutoff frequency determined by the beampipe radius at the if cavity nose). These narrow band, high- $Q$ resonance peaks correspond to wakefields that ring in the cavity for a large number of rf cycles, thus coupling each bunch to the other in the string of bunches in the accelerator. The resulting coupled-bunch motion may be linearly unstable if the beam intensity is high enough. Impedances presented to the beam by these parasitic modes of the cavity can be suitably represented by the frequency-dependent impedance of slightly damped, high- $Q$ resonators. The impedance of each longitudinal parasitic mode is given by

$$
Z_{\|}(\omega)=\frac{R_{S}}{1+i Q\left(\omega / \omega_{R}-\omega_{R} / \omega\right)}
$$

and that of each transverse parasitic mode by 


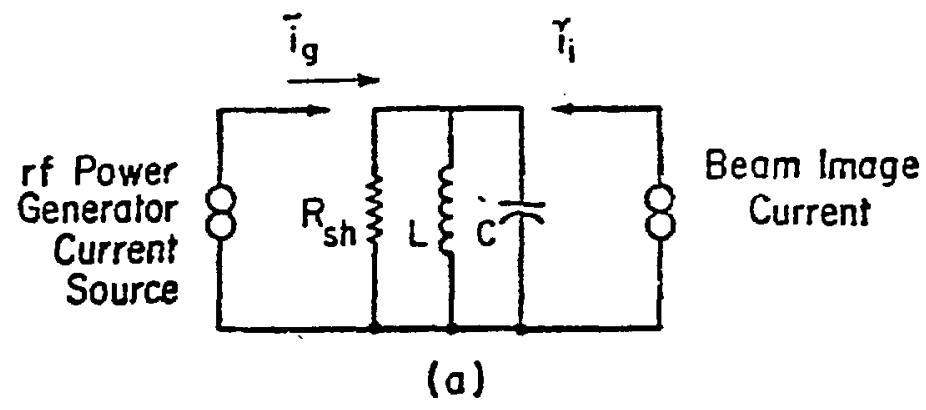

Fig. 12. Lumped RLC Circuit representing accelerating system being driven by two current sources, generator and beam. 


$$
Z_{\perp}(\omega)=\frac{\omega_{R}}{\omega} \frac{R_{T}}{1+i Q\left(\omega / \omega_{R}-\omega_{R} / \omega\right)}
$$

where $\omega_{R}$ is the resonance frequency, $Q$ is the quality factor, and $R_{S}\left(R_{T}\right)$ is the shunt impedance of the longitudinal (transverse) parasitic mode, expressed in ohms (ohms/meter). In order to avoid coupled bunch instabilities, those higher-order modes have to be measured and properly damped.

\section{BEAM MANIPULATION AND BEAM CONTROL}

So far we have described the generation of radio-frequency voltage in the accelerating cavity and the ideal particle behavior in a synchrotron. To make al synchrotron useful, more if manipulations are needed besides simple acceleration. For example, the synchrotron usually receives the beam from the linac which has a much higher radio-frequency, typically $200 \mathrm{MHz}$. For all practical purposes, the injected beam has a continuous longitudinal distribution. To accelerate such a beam, a process to create a bunched distribution compatible with the bucket structure of the rf system has to be found. One adopted method is adiabatic of capture.

\subsection{RF CAPTURE, BUNCHING, AND DEBUNCHING}

Ideally, the synchronous phase is set to zero to create a stationary bucket, because then all phases are stable and any particle within the bucket will be trapped. The acceleration voltage is increased slowly, the bucket height grows, and more and more of the particles with larger energy spread are trapped, as illustrated in Fig, 13.

Assuming that the energy spread of the injected linac beam is $W_{L}$, the phase-space area of the ribbon beam in one of period is given by 


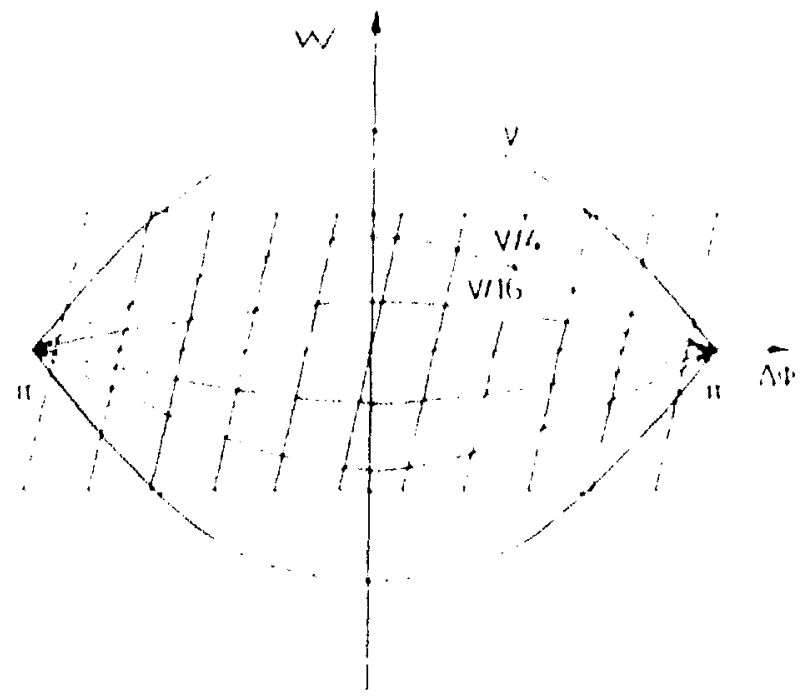

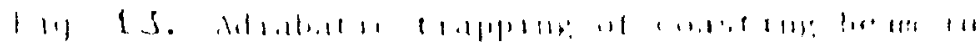

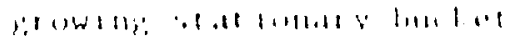

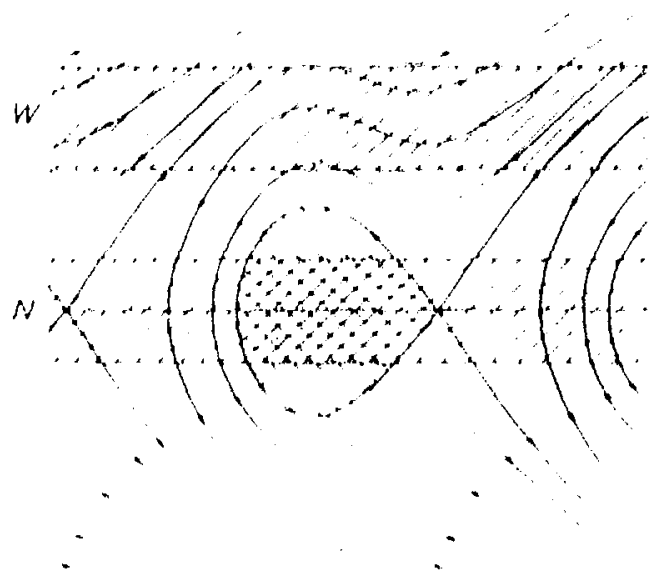

(I)

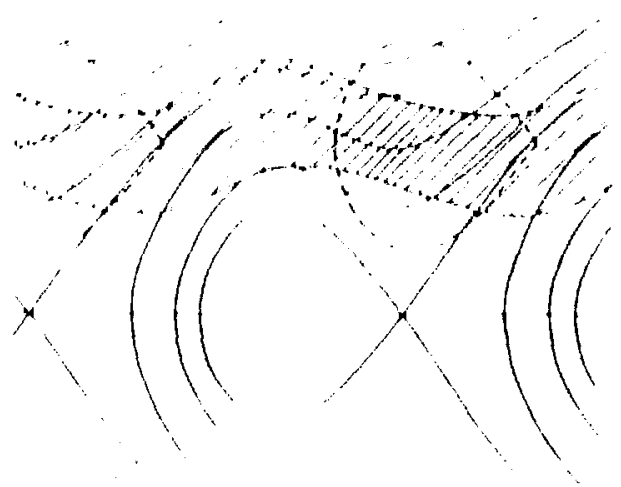

b)

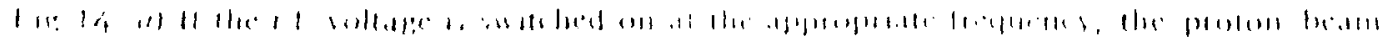

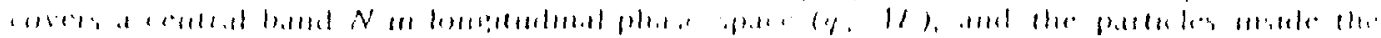

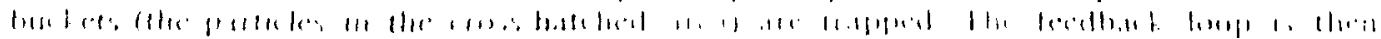

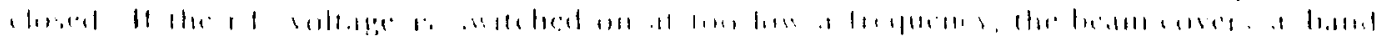

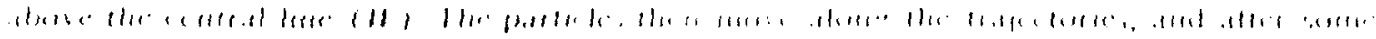

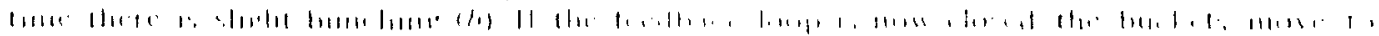

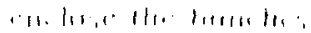




$$
A_{S}=4 W_{L} \pi
$$

From Eq. (33) it can be shown that the phase-space area of a stationary bucket with bucket height $W_{b}$ is

$$
A_{b}=8 W_{b}
$$

Exercise: Prove Eq. (4.2).

Usually, one figure of merit in judging how much if voltage is needed for adiabatic capture is given by the condition that area (4.2) equals area (4.1), in other words

$$
W_{b}=\frac{\pi}{2} W_{L}
$$

According to this criterion, the maximum capture efficiency is about $74 \%$, which is just the overlap integral of the stationary bucket with the linac beam. To get better capture efficiency, larger voltage must be used.

But how slow is slow? What constitutes an adiabatic variation of if voltage? One way to quantify this concept is due to Lilliequist and Symon. They assume the condition that the fractional increment $d A / A$ in bucket area within a time interval $d t$ is always proportional to the fractional time increment $d t / \tau_{p}$, where $\tau_{p}$ is the phase oscillation period at that instant, i.e.

$$
\frac{d A}{A}=\alpha \frac{d t}{\tau_{p}}
$$

We know that

$$
A=C_{1} V^{1 / 2}, \quad \tau_{p}=C_{2} V^{-1 / 2},
$$


so that Eq. (4.4) becomes

$$
\begin{gathered}
\frac{1}{2} \frac{d V}{V}=\frac{\alpha}{C_{2}} V^{1 / 2} d t, \\
V(t)=\frac{V_{2}}{\left[\left(\frac{V_{2}}{V_{1}}\right)^{1 / 2}-\alpha \frac{t-t_{1}}{\tau_{12}}\right]^{2}},
\end{gathered}
$$

where $t_{1}$ and $V_{1}$ are the initial time and voltage and $V_{2}$ and $\tau_{p 2}$ are the final voltage and the corresponding phase oscillation period. The degree of adiabaticity is specified by $\alpha$. If $\alpha \ll 1$, the process is adiabatic. For $\alpha \gg 1$, the process corresponds to a fast turn-on.

Actual practice for highest efficiency of if capture does not necessarily favor the adiabatic capture process. There are many reasons. One is that the real adiabatic process takes a long time and therefore the magnetic imperfection resonances can destroy the beam. Another reason is that, in the early stage, the voltage is purposely kept low. For a high intensity proton beam, the space charge created by neighboring particles will blow the beam outside the bucket. Therefore, for a high intensity beam, we have to start capture at moderate voltage. Sometimes the best capture efficiency comes from off-momentum capture, as shown in Fig. 14.

Another interesting phenomenon is the process that is the reverse of bunching, i.e. debunching. The easiest way to debunch a beam is to turn off the if voltage and let the beam drift. The height of the bucket will stay constant, anci the particles just drift along lines parallel to the $\phi$ axis at a rate such that they come into contact with adjacent buckets and finally smear into a continuum after a given time $t$, as shown in Fig. 15.

It is quite straightforward to estimate the debunching time. By definition, the time in which the front of one bunch reaches the tail of an adjacent bunch 


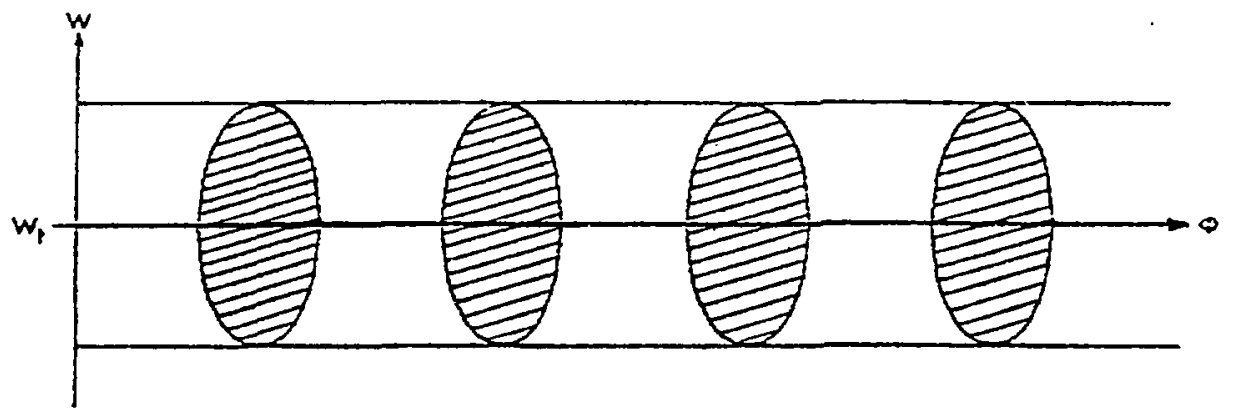

(a)

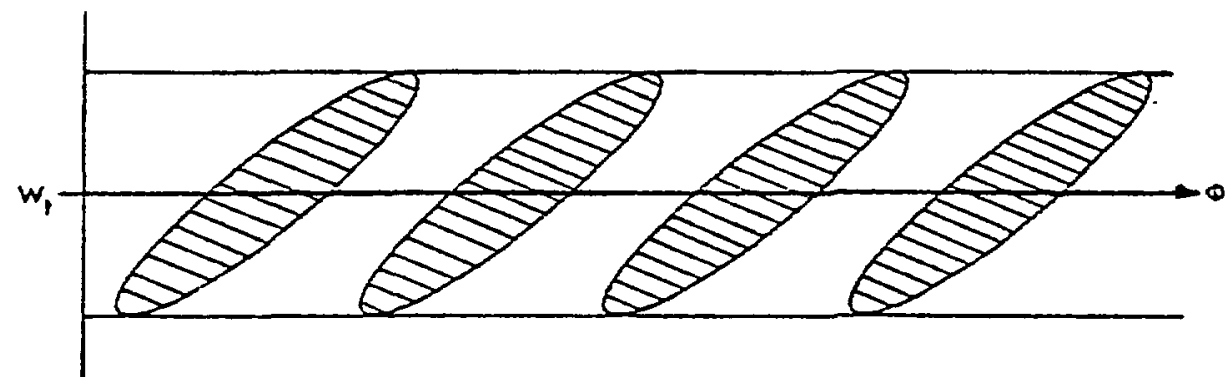

(b)

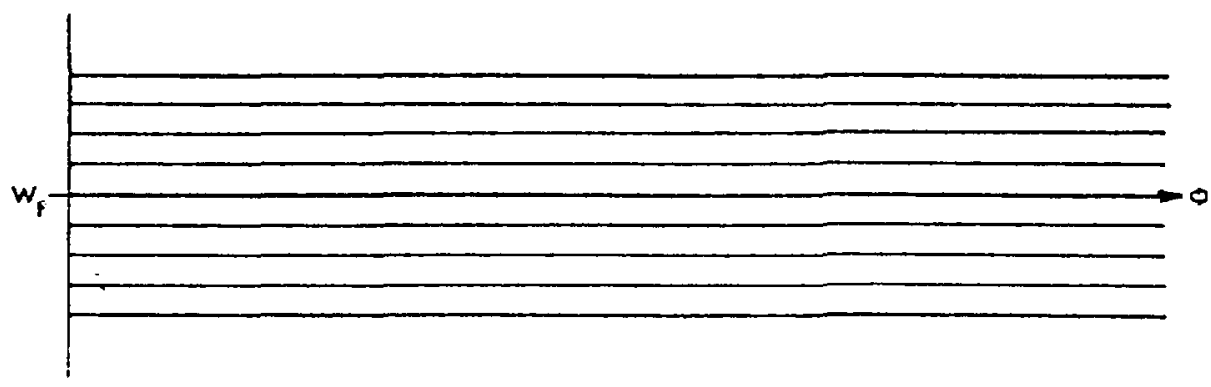

(c)

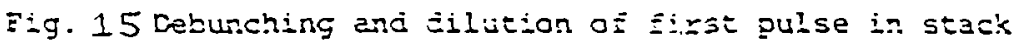


is the debunching time. We know that the distance between the boundaries of two bunches is

$$
\Delta S=\frac{\pi-\Delta \phi}{2 \pi h} 2 \pi R
$$

The difference in revolution period between the fastest and the slowest particles (top and bottom) is given by

$$
\Delta T_{\text {rev }}=\frac{1}{f_{\text {rev }}+\Delta f}-\frac{1}{f}=\frac{\Delta f}{f_{\text {rev }}}=\eta \frac{\Delta p}{p}
$$

and the corresponding difference in velocity is

$$
\Delta v=2 \pi R \Delta f=2 \pi R f_{\text {rev }} \eta \frac{\Delta p}{p}
$$

Therefore

$$
t_{d b}=\frac{\Delta S}{\Delta v}=\frac{\pi-\Delta \phi}{2 \pi f_{\text {rev }} h \eta \Delta p / p} .
$$

Debunching is usually used for slow extraction in fixed-target experiments where the event rates have to be limited, or for changing harmonic number during the acceleration process. Ideal debunching should result in a beam with a uniform distribution, but, seen in from Fig. 15, it takes a long time to produce a uniform beam since the beam is denser in the center than at the boundary. Worse yet, reality is more complicated than the simple model suggests. One instance is that the beam-induced voltage at the gap of the acceleration cavity tends to (self) bunch the beam. A shorting switch is usually provided to prevent that from happening. Results produced by the ESME tracking program on bunching and debunching are shown in Figs. 17, 18, and 19 for comparison. 

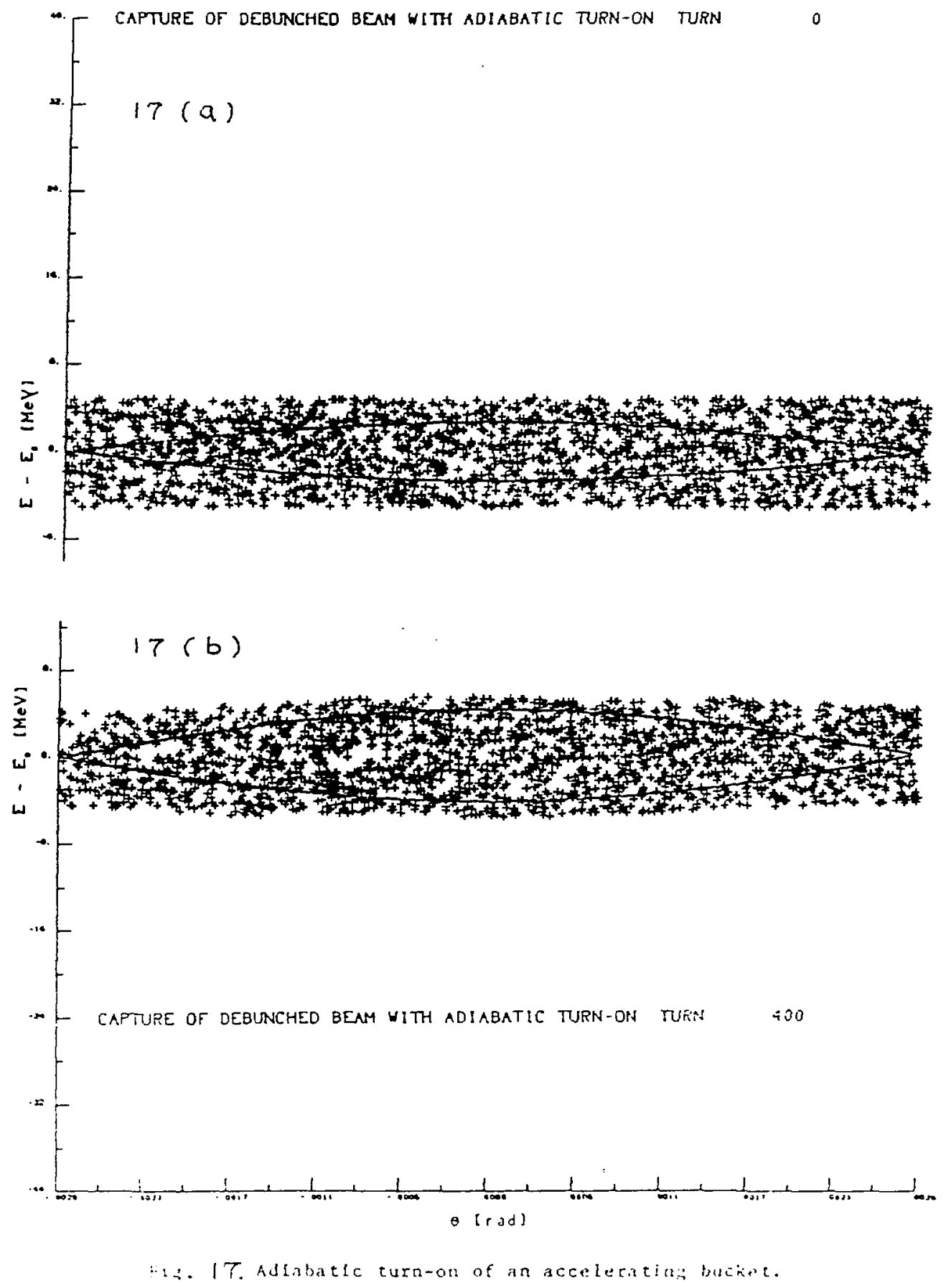

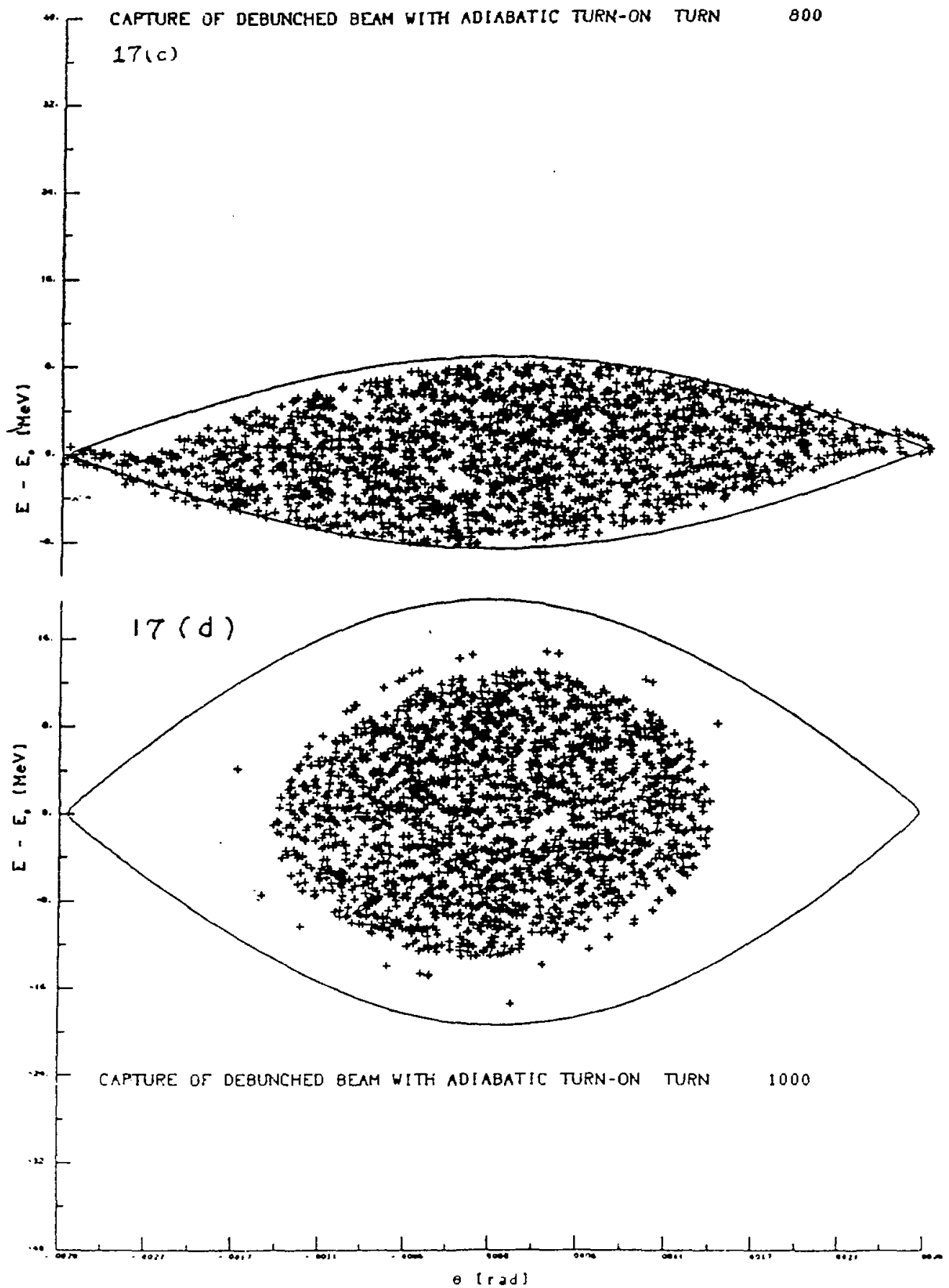

Fig。 $17 c, 17 d$ 。 

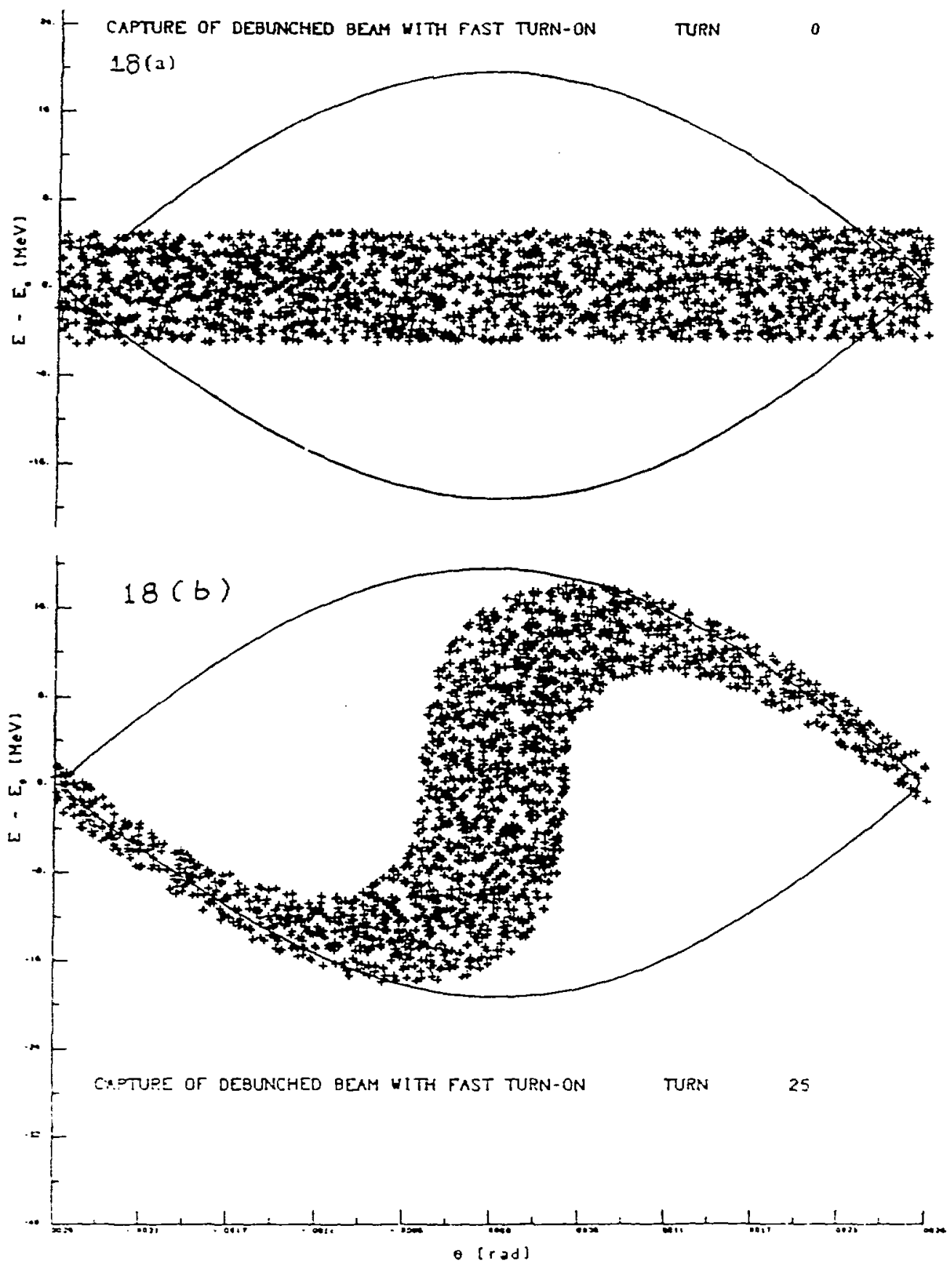

is. 18. Sudden turn-oil of an accelerating bucret. 


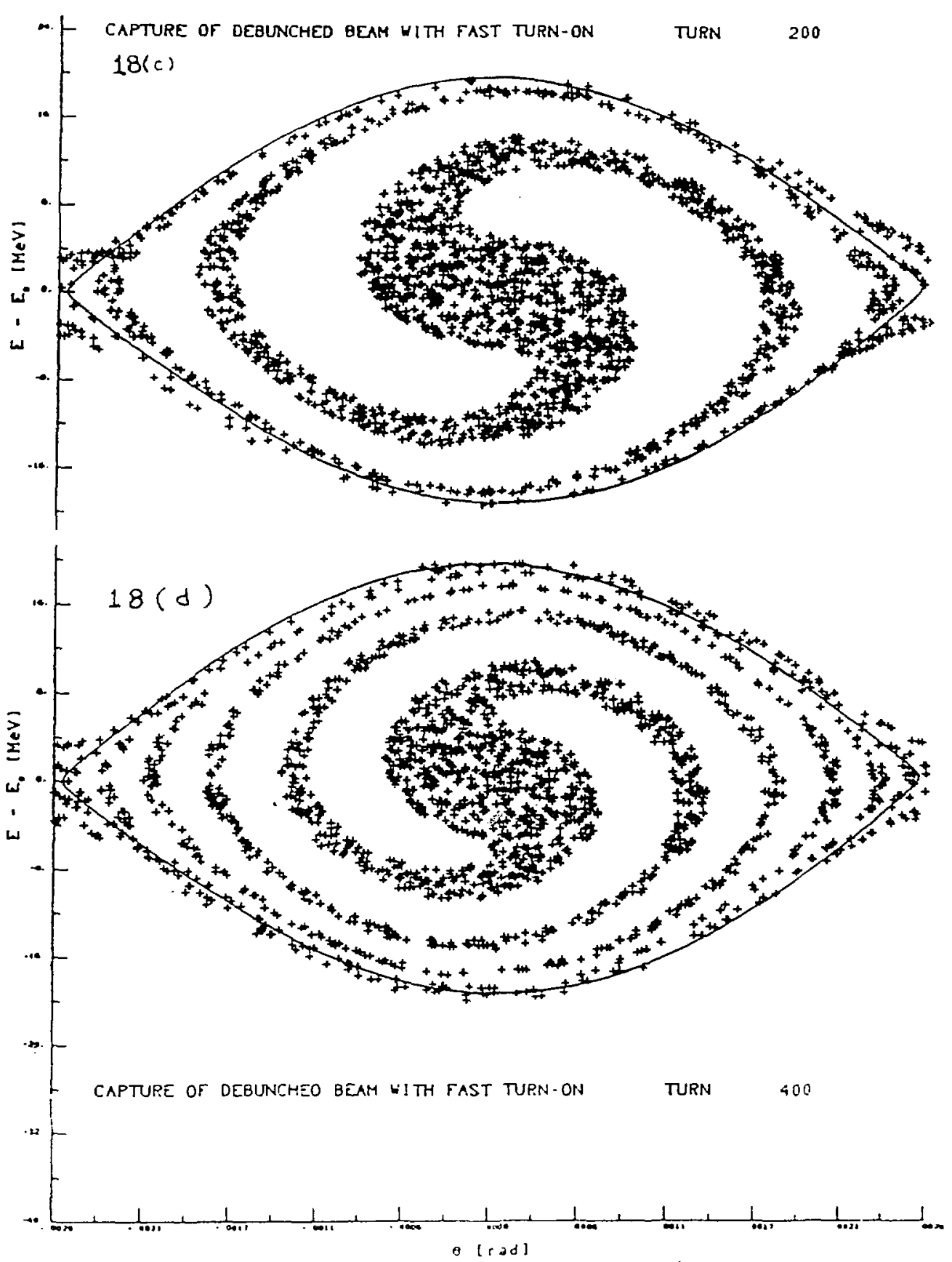

Fig, 18c, 18d。 

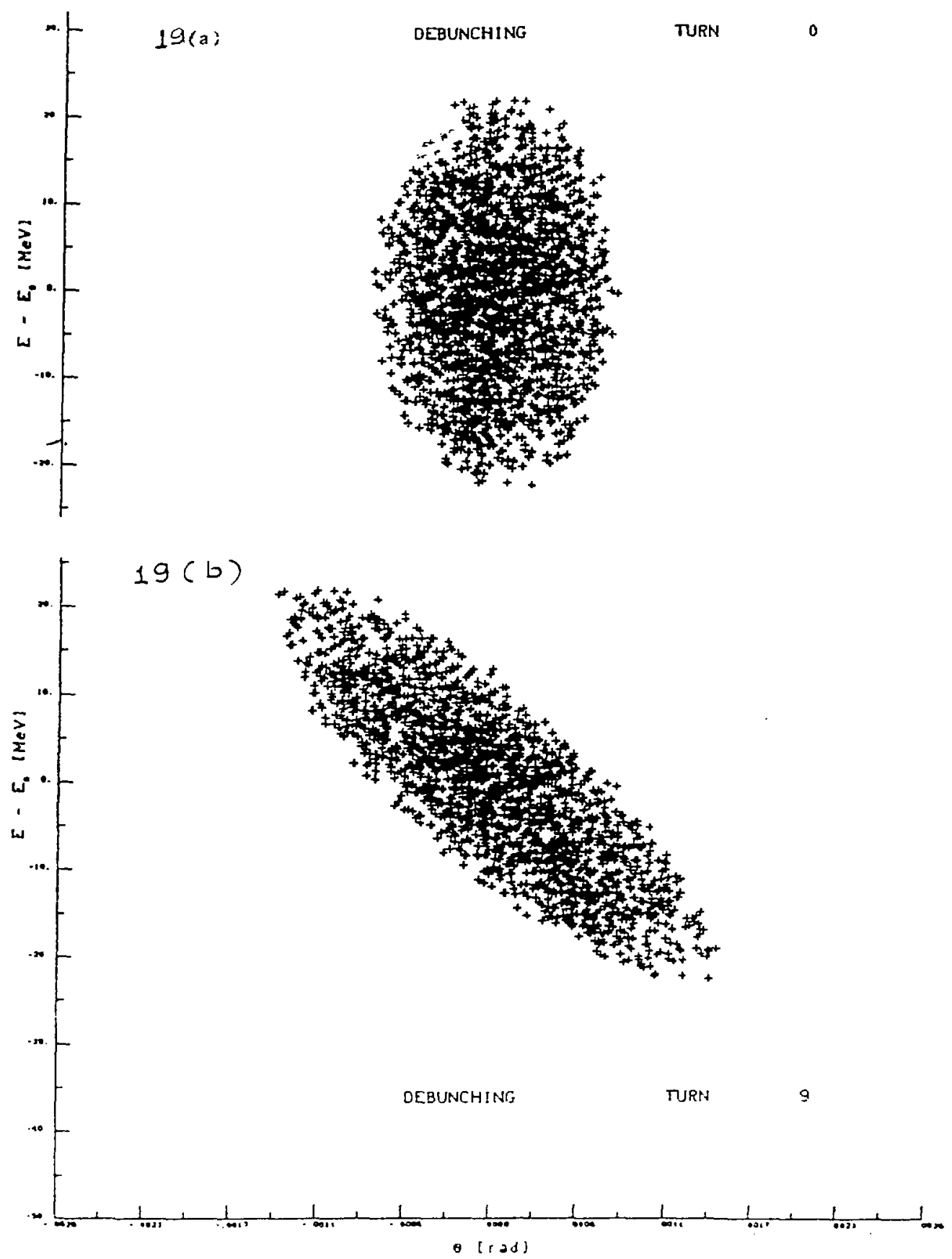

rig. 19. Debunching by drifting and by a buctiret. 


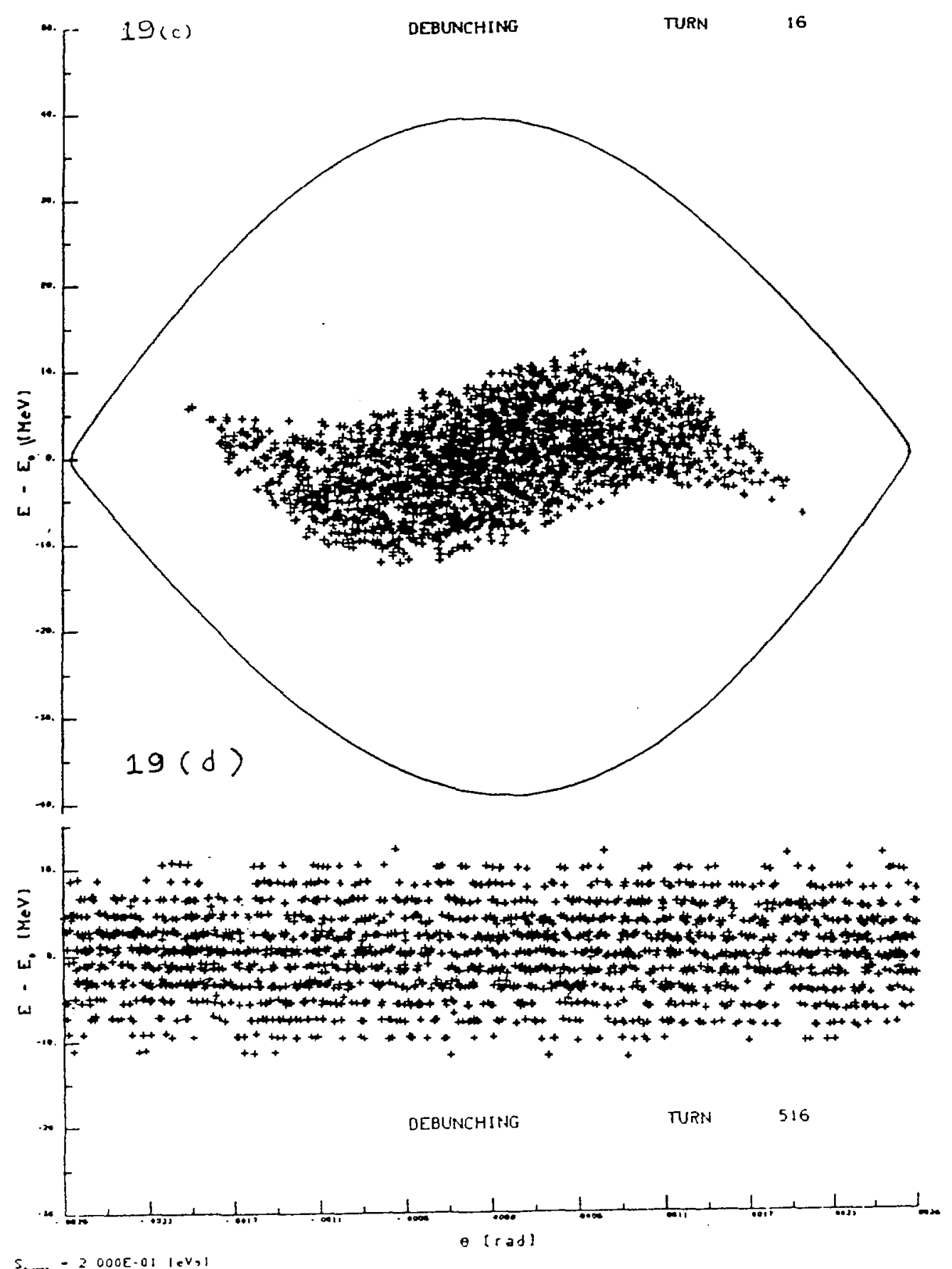

Fig。19c, 19d. 
In the design study of the AGS booster project ${ }^{13}$, it has been found that under intense space charge effects, the beam tends to blow up in the first few turns after injection. It is therefore important in the simulation study to build up the charge in the ring turn by turn from the linac, like in the actual process, instead of having the full intensity appear at once. Another important phenomenon is the fact that those beam particles that are close to the unstable fixed points are lost at the end of the capture process. To minimize this effect, a chopped beam from the linac can be used as suggested in Ref. 13.

\subsection{SYNCHRONOUS TRANSFER AND PHASE-SPACE MATCHING}

In transferring a beam of particles from one synchrotron to another, the most important considerations are efficiency and phase-space dilution. To preserve the longitudinal emittance of the beam, the phase-space ellipses of the two synchrotrons should have the same shape and orientation, in other other words, be matched. One way to do this is to make the ratio $\hat{W} / \Delta \hat{\phi}$ identical for the two accelerators,

$$
\left(\frac{\hat{W}}{\Delta \hat{\phi}}\right)_{\text {Booster }}=\left(\frac{\hat{W}}{\Delta \hat{\phi}}\right)_{A G S}
$$

Since

$$
\frac{\hat{W}}{\hat{\Delta} \phi}=\left(\frac{\beta^{2} E_{0} h \Omega_{\circ}^{2} e \cos \phi_{\circ}}{2 \pi \eta}\right)^{1 / 2}
$$

therefore,

$$
\left(\frac{h \Omega_{\mathrm{o}}^{2} \cos \phi_{\mathrm{o}}}{\eta}\right)_{B \text { ooeter }}=\left(\frac{h \Omega_{\mathrm{o}}^{2} \cos \phi_{\mathrm{o}}}{\eta}\right)_{A G S}
$$

If in addition $\left(\cos \phi_{0}\right)_{B o o s t e r}=\left(\cos \phi_{0}\right)_{A G S}$, the trajectories have identical 
shapes in the two accelerators even for large amplitudes, and therefore a perfect match can be obtained for bunches of any length.

The matched condition is often achieved by adiabatically varying the voltage in the first, lower energy accelerator. But, the beam loading instability may make it difficult to control the phase and the amplitude of the rf voltage when it is low. With bunch rotation, matched bunches can be created in the first machine at a much higher voltage and in a shorter period, as illustrated in Fig. 20.

\subsection{PERTURBED SYNCHROTRON OSCILLATIONS}

We have shown that, in ideal acceleration, the phase of a non-synciaronous particle behaves like a SHO, satisfying

$$
\frac{\partial^{2} \Delta \phi}{\partial t^{2}}+\Omega^{2} \Delta \phi=0
$$

In the presence of errors in the magnetic guiding field, the revolution frequency, and the voltage amplitude, however the equation of motion becomes

$$
\frac{\partial^{2} \Delta \phi}{\partial t^{2}}+\Omega_{\circ}^{2} \Delta \phi=-h \alpha \omega \frac{d}{d t}\left(\frac{\delta B}{B}\right)+\frac{d}{d t}(\delta \omega)+\Omega_{\circ}^{2} \tan \phi_{\circ} \frac{\delta V}{V}=f(t)
$$

This shows that $\delta B / B$ is important for an accelerator with a large $\alpha$ (weak focusing) and that a field ripple can be compensated by a corresponding frequency swing.

Now let us look at the possible sources of errors and their effect on the synchrotron oscillations.

(a) General perturbation $f(t)$, then 
(a)

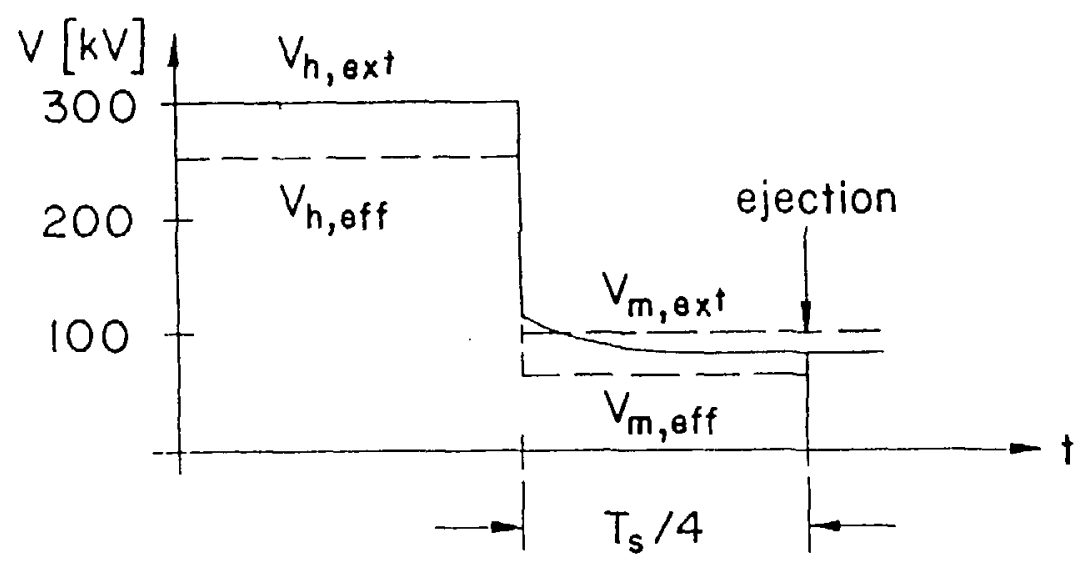

Fig. 20. Bunch rotation matching

(b)

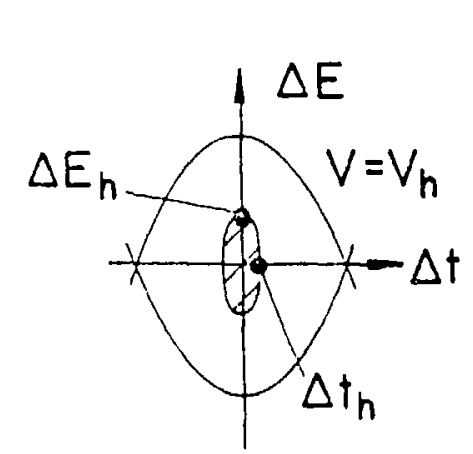

Matched bunch

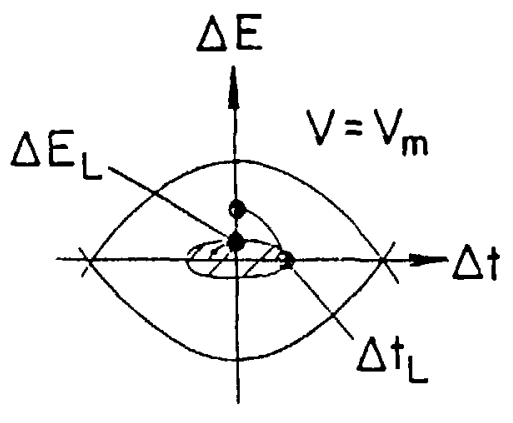

After turn in mismatched bucket Fig. 20 .

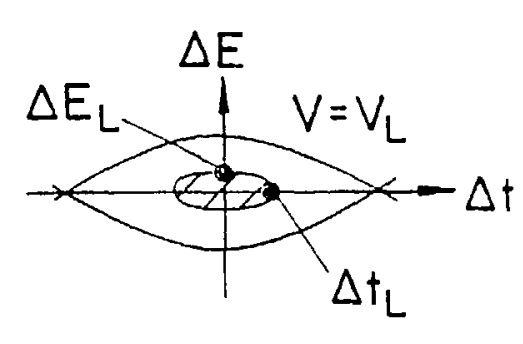

Equivalent matched bucket 


$$
\Delta \phi(t)=\frac{1}{\Omega_{s}} \int_{t_{0}}^{t} f(\lambda) \sin \Omega_{s}(t-\lambda) d \lambda .
$$

This is a solution through Green's function and can be verified by substitution into Eq. (4.16).

(b) Sinusoidal perturbation $f(t)=$ asinwt, then

$$
\Delta \phi(t)=\frac{a}{\Omega_{a}^{2}-\omega^{2}} \text { sinwt. }
$$

This is a forced oscillation when away from resonance. If at resonance, $\omega=\Omega$, and the solution becomes

$$
\Delta \phi(t)=\frac{a t}{2 \Omega,} \sin \left(\Omega, t-\frac{\pi}{2}\right) .
$$

The phase then increases linearly with time without limit.

Exercise: Prove Eqs. (4.18) and (4.19).

(c) Passage through a resonance:

$$
f(t)=\operatorname{asin} \int_{t_{0}}^{t} \omega(\lambda) d \lambda
$$

and

$$
\omega\left(\lambda_{\circ}\right)=\Omega_{.} .
$$

We can expand the perturbing frequency around the syachrotron oscillation frequency $\Omega$, such that

$$
\omega=\Omega_{\bullet}+\dot{\omega} t+\ldots
$$

then 


$$
f(t)=\operatorname{asin} \int_{0}^{t} \omega d \lambda=\operatorname{asin}\left(\Omega_{s} t+\frac{1}{2} \dot{\omega} t^{2}\right),
$$

and again the general solution (4.17) gives

$$
\Delta \phi(t)=-\frac{a}{\Omega_{s}}\left(\frac{\pi}{2 \dot{\omega}}\right)^{1 / 2} \cos \left(\Omega_{s} t+\frac{\pi}{2}\right) .
$$

This is the additional oscillation induced by passage through a resonance. Any external perturbation with a frequency equal to a multiple of $\Omega$, should also be avoided.

In addition to eliminating the sources of perturbations, feedback loops are usually provided in an if system to keep the orbit, frequency, and voltage deviations within tolerances. The principles of $\mathrm{rf}$ control will not be discussed here; those interested should consult Refs. 14, 15, and 16.

\subsection{BEAM LOADING AND ROBINSON INSTABILITY}

The behavior of an rf cavity can be approximated by an equivalent circuit, as shown in Fig. 21a, which shows a parallel resonant RLC circuit driven by the rf power source and the beam image current. If the beam intensity is small $\left(i_{i} \ll i_{g}\right)$, the phasor diagram of the cavity is shown in Fig. $21 \mathrm{~b}$, where

$\vec{i}_{b}=$ beam current (reference),

$\overrightarrow{i_{i}}=$ induced current $=-\overrightarrow{i_{b}}$,

$\overrightarrow{i_{g}}=$ generator current,

$\vec{V}_{g}=$ gap voltage excited by $\vec{i}_{g}$, and

$\theta=$ equivalent lead of $\vec{i}_{g}$ with respect to $\vec{i}_{b}$.

In the presence of beam current, the induced current will produce an induced voltage at the gap $\left(\vec{V}_{b}\right)$ making the total voltage across the cavity deviate from a design amplitude and phase, 

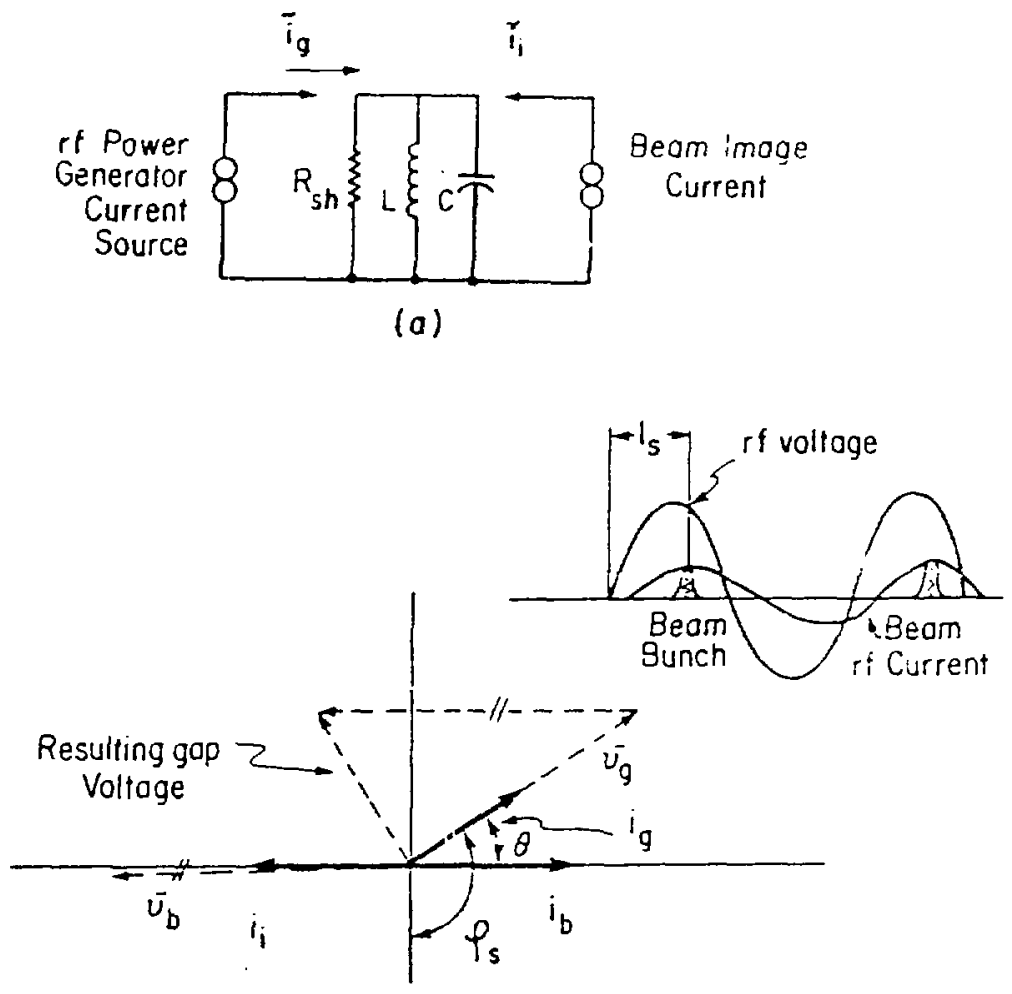

(b)

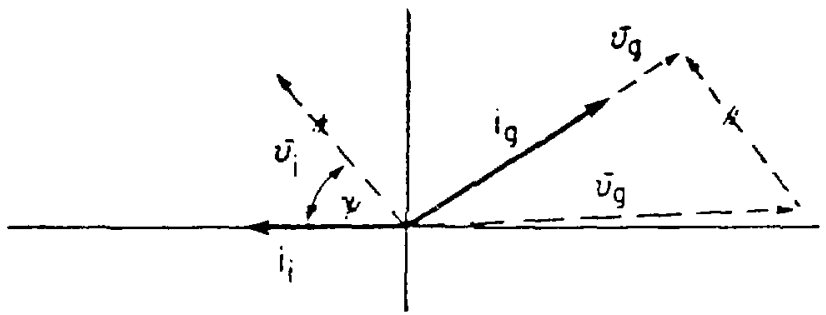

(c)

Fi;. 2:. (a) Lumped RLC Circuit rapresenting acceleratins sostem being driven by two current sources, generater and beam.

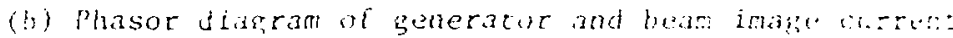

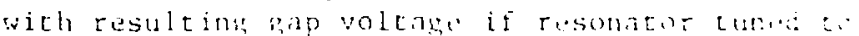
resonames.

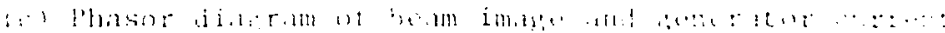

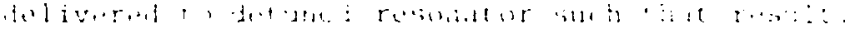

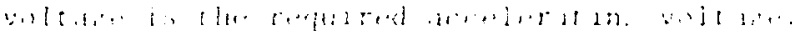




$$
\vec{V}_{c}=\vec{V}_{g}+\vec{V}_{b}
$$

This phenomenon is called beam loading, and there are two possible solutions to alleviate the problem:

1. Feedforward by providing additional current through the power amplifier to cancel the induced current, such that $\vec{i}_{f}=-\vec{i}_{i}=\vec{i}_{b}$.

2. Detune the cavity, in which case the detuning angle is given by

$$
\Psi=\tan ^{-1}\left(2 Q \frac{\Delta \omega}{\omega_{r}}\right)
$$

The resulting impedance is

$$
Z=R_{s h} \cos (\Psi) e^{-j \Psi}
$$

If the resonance frequency is detuned to below the excitation frequency, the impedance will be partially capacitive. Then, the voltage developed will lag behind the exciting current in time. The detuning angle and the generator current are now adjusted so that the resultant capacity voltage has correct magnitude and angle. When this is done, the rf voltage appearing across the generator is in phase with the generator current and the load appears real to the generator.

Let $\vec{i}_{0}=i_{0} e^{j \theta} \approx$ generator current required to develop the acceleratio. voltage in (real) $R_{s h}$ in the absence of beam. Then the condition that detuning of the cavity giving identical voltage across the cavity is

$$
\vec{V}_{c}=i_{0} R_{g h} e^{j \theta}=\vec{V}_{g}+\vec{V}_{i}=i_{g} e^{j \theta} R_{s h} \cos (\Psi) e^{-j \Psi}-i_{i} e^{j \theta} R_{s h} \cos (\Psi) e^{-j \Psi}
$$


Equating the real and imaginary parts of Eq. (4.27) separately, we get explicit expressions for the required detuning angle and generator current in terms of the beam current

$$
\begin{aligned}
& i_{g}=i_{\circ}+i_{i} \cos \theta \\
& \tan \Psi=\frac{i_{i}}{i_{0}} \sin \theta
\end{aligned}
$$

This detuning will restore the cavity voltage to proper amplitude and angle for beam acceleration. The limit on the detuning angle is set by the constraint $\Psi \leq \pi / 2$, as shown in Fig. 21c.

Now let us examine the stability condition of the synchrotron oscillations of the beam centroid under the detuned condition. Assuming that the initial phase offset of the bunch is $\eta$, as shown in Fig. 22, the voltage seen by the beam will be

$$
V_{a c c}=V_{g} \cos (\theta-\eta) \approx V_{g} \cos \theta+\eta V_{g} \sin \theta=V_{g} \sin \phi_{\circ}+\eta V_{g} \cos \phi_{\circ}
$$

Another source of voltage change is the displacement of the beam phasor resulting in an additional small excitation current $\vec{i}_{d}$, which generates an additional voltage $\vec{V}_{d}$,

$$
\begin{gathered}
\vec{i}_{d}=j \eta \vec{i}_{i}=-j \eta i_{i}, \\
\vec{V}_{d}=-j \eta i_{i} Z=\eta Z\left(\frac{i_{i}}{i_{o}}\right) \cos (\Psi) e^{-j(\Psi+\phi / 2)}
\end{gathered}
$$

Hence, the corresponding additional voltage for acceleration is 


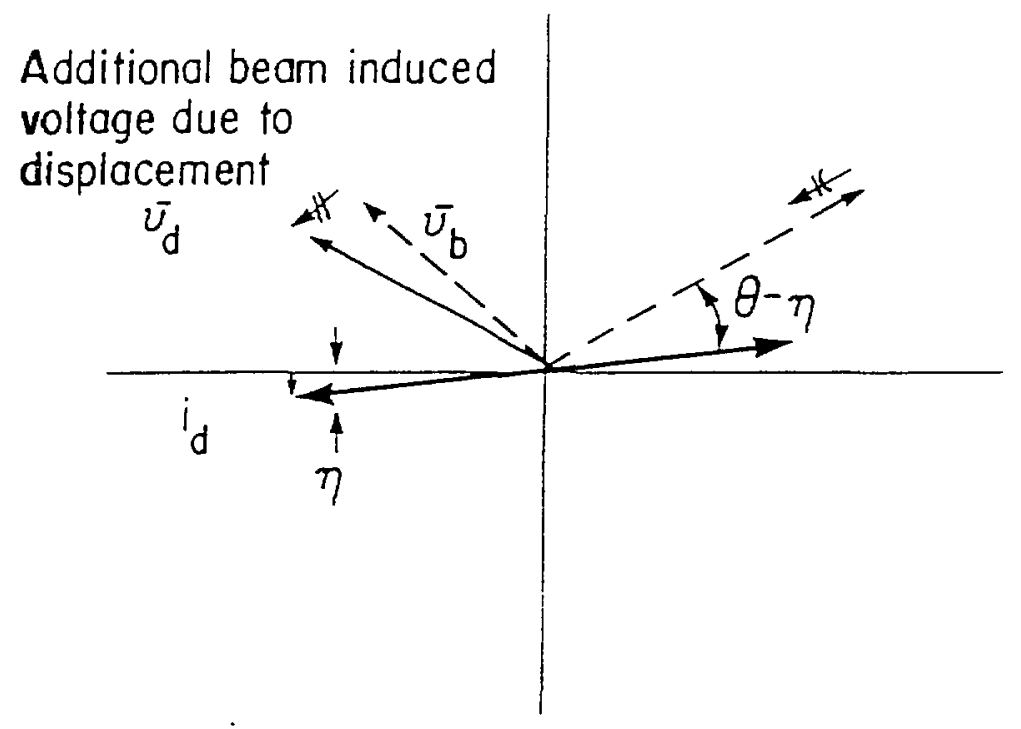

Fig. 2.2. (a) Beam and image current displaced from equilibriu: position by small angle $\eta$ resulting in degradazisa of accelerating voltage. 


$$
v_{a c c}=\eta V_{g}\left(\frac{i_{i}}{i_{0}}\right) \cos (\psi) \cos \left(\theta+\Psi+\frac{\pi}{2}\right)=-V_{g} \eta\left(\frac{i_{i}}{i_{0}}\right) \sin \Psi \cos \Psi
$$

making the total voltage

$$
V_{\text {total }}=V_{g} \sin \phi_{\circ}+\eta V_{g} \cos \phi_{\circ}\left(1-\frac{i_{i}}{i_{\circ}} \frac{\sin \Psi \cos \Psi}{\cos \phi_{\circ}}\right)
$$

The second term determines the synchrotron oscillations under beam loading with detuning. It can be seen that $i_{i}$ reduces the available voltage for damping the initial phase error. The limiting condition for stability is

$$
2 \frac{i_{i}}{i_{\circ}} \cos \phi_{\circ} \geq \sin 2 \Psi \geq 0
$$

which the is well-known Robinson criterion and is consistent with our previous condition that the detuning angle should satisfy

$$
\Psi \leq \frac{\pi}{2}
$$

\section{HYGH INTENSTTY EFFECTS}

If the charge density in a bunch becomes large, the Coulomb repulsive force between the particles can no longer be neglected. The space-charge force will cause a tune shift of the betatron oscillations in both horizontal and vertical planes. Longitudinally, it will modify the focusing effect of the external if forces. In the following, we will discuss the effect of space charge on the longitudinal motion of the particles in a synchrotron ${ }^{18}$.

\subsection{SPACE-CHARGE FORCE}


To estimate the importance of the space charge-force, we will make a comparison between the force constant associated with the longitudinal spacecharge forces and that due to the if voltage. The rf system accelerates the particles by $\hat{V} \sin \phi_{\mathrm{o}}$ volts per turn; the corresponding field is

$$
E=\frac{e \hat{V}}{2 \pi R} \sin \phi_{\circ}
$$

The focusing force is given by the azimathal variation of the field:

$$
\left(\frac{d E}{d s}\right)_{r f}=-\frac{h}{R} \frac{d E}{d \phi}=-\frac{e h V}{2 \pi R^{2}} \cos \phi_{0} .
$$

To simplify the calculation, we assume a longitudinal charge distribution in the bunch that will make the space-charge force linear, i.e.

$$
\lambda(s)=\frac{3}{4} \frac{N e}{R \hat{\theta}}\left[1-\left(\frac{h s}{R \hat{\theta}}\right)^{2}\right],
$$

where $s$ is the distance along the circumference. This expression represents a parabolic shape of half length $\hat{\theta}$ rf radians. Assuming the bunch is of circular extent $a$ within a circular vacuum chamber of radius $b$, the voltage seen by the beam is given by

$$
V(s)=\frac{g_{\circ}}{4 \pi \epsilon_{\circ}} \lambda(s)
$$

where

$$
g_{\circ}=1+2 \ln (b / a)
$$

Hence, the longitudinal space-charge feld is

$$
E(s)=\frac{d V(s)}{d s}
$$


and the focusing force becomes

$$
\left(\frac{d E}{d s}\right)_{a c}=\frac{3}{2 \lambda^{2}} \frac{g_{\circ}}{4 \pi \epsilon_{\circ}} \frac{h^{2}}{R^{3}} \frac{N e}{\hat{\theta}^{3}} .
$$

The ratio between $(d E / d s)_{d c}$ and $(d E / d s)_{r f}$ is a measure of the strength of space charge. Let us define the ratio between these two forces as $\eta_{x c}$, then

$$
\eta_{s c}=\left(\frac{d E}{d s}\right)_{e c} /\left(\frac{d E}{d s}\right)_{r f}=\frac{3}{2} \frac{h}{R \lambda^{2}} \frac{2 \pi}{e V\left(-\cos \phi_{\circ}\right)} \frac{g_{\circ}}{4 \pi \epsilon_{\circ}} \frac{N e}{\theta^{3}} ;
$$

$\eta_{s c} \approx 1.8$ for the AGS at $N=10^{13}$. This reduction in longitudinal focusing can actually cause beam blowup or beam loss. With the booster addition, the AGS intensity will increase to $N=6 \times 10^{13}$ and $\eta_{\text {ac }}$ will be about 10 . Something has to be done to save the beam.

\section{$5.2 \gamma_{t r}$-JUMP AT TRANSITION}

It is convenient to view particle behavior at transition in terms of mass. Below $\gamma_{t r}$, as a particle is accelerated, its angular velocity increases: the harder it is pushed, the faster it goes. Above $\gamma_{t r}$, the situation is reversed: pushing decelerates; the particle behaves as if it had a negative mass. In this case, the apparent mass of a particle as a function of energy behaves as shown in Fig. 23.

Now, recall that the bunch length is given by

$$
\Delta \phi=\left(\frac{-\eta \Omega_{\circ}}{P_{\mathrm{o}} R_{\mathrm{o}} \hat{V} \cos \phi_{\mathrm{o}}}\right)^{1 / 4},
$$

where now the focusing force should be replaced by an equivalent quantity including space-charge force such that

$$
\hat{V} \cos \phi_{\circ}-\left(\frac{d E}{d s}\right)_{e c} \leq \hat{V} \cos \phi_{\circ}, \quad \gamma \leq \gamma_{t r}
$$




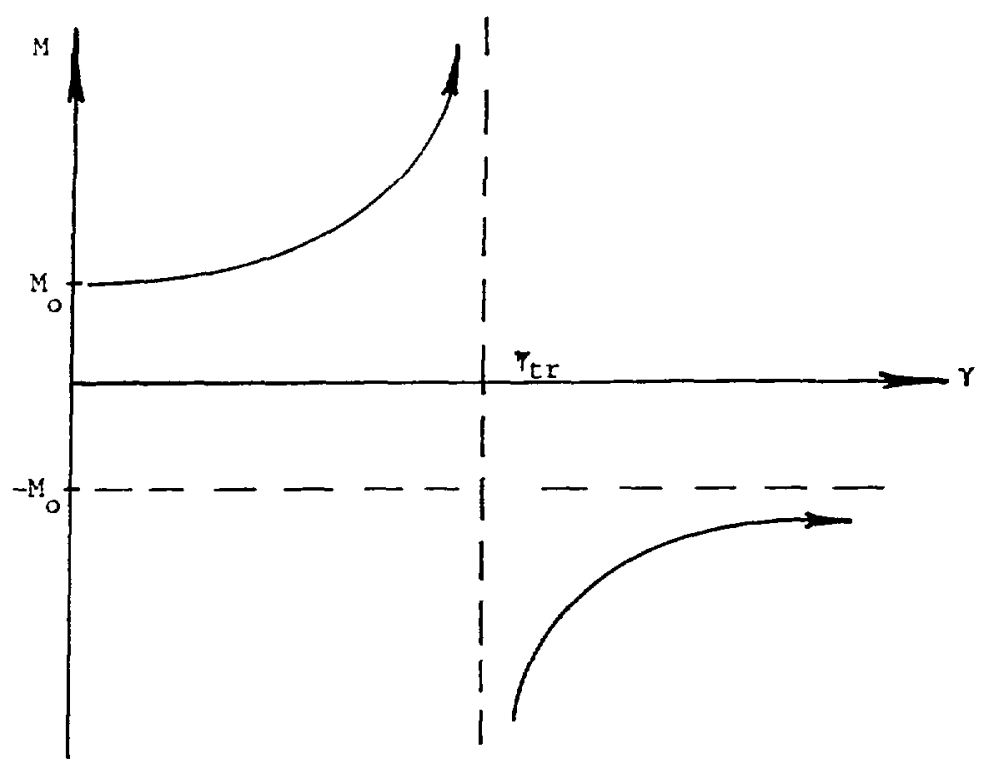

Fig. 23 The apparent mass of a proton in a synchrotion

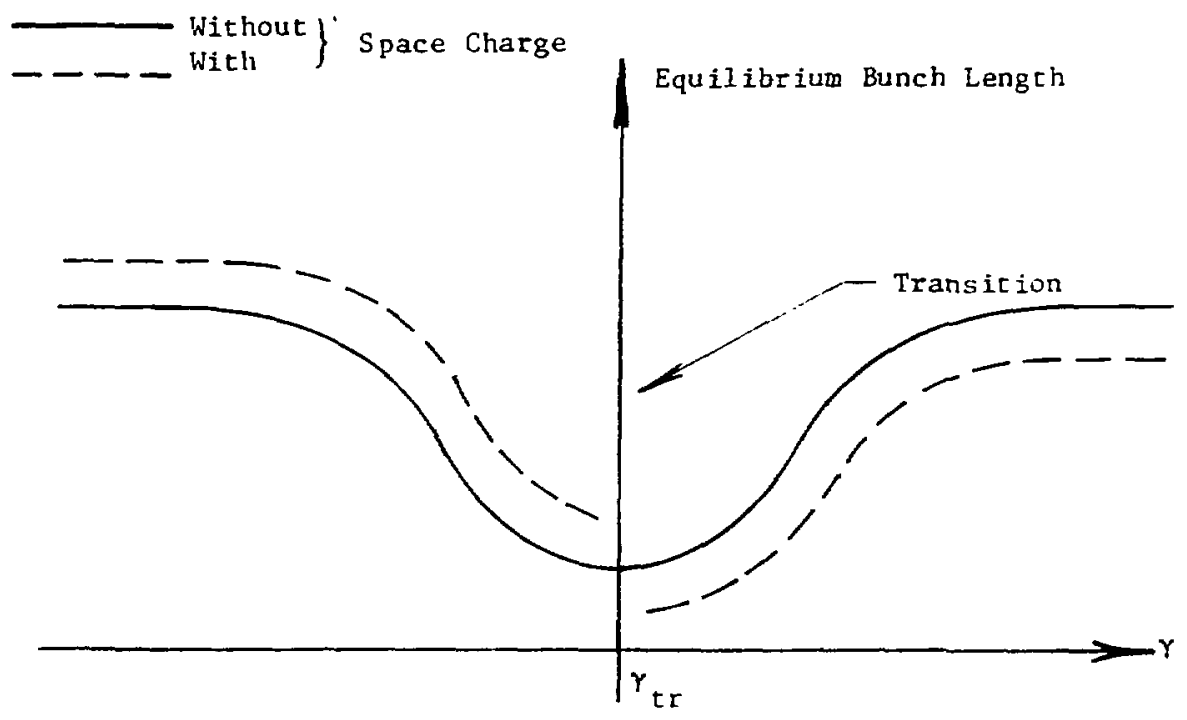

Fig. 24 The bunch shape mismatch of transition energy 


$$
\hat{V} \cos \phi_{0}+\left(\frac{d E}{d s}\right)_{e c} \geq \hat{V} \cos \phi_{0}, \quad \gamma \geq \gamma_{t r}
$$

The positive sign in Eq. (5.8b) represents the attractive force due to the negative mass effect. The net result is that the bunch length is different before and after transition, hence phase-space mismatch, as shown in Fig. 24.

Furthermore, at transition, the frequency spread of the synchrotron oscillations is small. Thus, just above transition, a situation exists in which growing oscillations can be excited; this is usually called the negative mass instability. The size of these oscillations, and the resulting emittance blowup, depends on the beam intensity and the time spent in the regime with little damping. Two approaches can be taken to reduce these transition losses:

1. Artificial enlargement of the bunch area before transition to reduce the space-charge force.

2. Minimization of the time spent in the unstable region by varying $\gamma_{t r}$ near transition.

The second method is called $\gamma_{t r}$-jump and was first proposed by Teng and Hardt.

A quadrupole pair separated by $1 / 2$ betatron wavelength and configured as doublets can alter the $\gamma_{t r}$ of a synchrotron without affecting its tune. By pulsing such quadrupoles, the time spent in the unstable region during transition can be reduced as shown in Fig. 25.

One figure of merit is the crossing-speed enhancement factor, $f^{\prime}$. The relationship between $f^{\prime}$, the bunch area, and the intensity limit for the $A G S^{19}$ is shown in Fig. 26. Without $\gamma_{t r}$-jump, the AGS can pass through transition without visible beam blowup at $N=2 \times 10^{12}$ if the bunch area is $1 \mathrm{eV}$-sec. With a $\gamma_{t r}$-jump system having enhancement factor $f^{\prime}=30$, with the same 


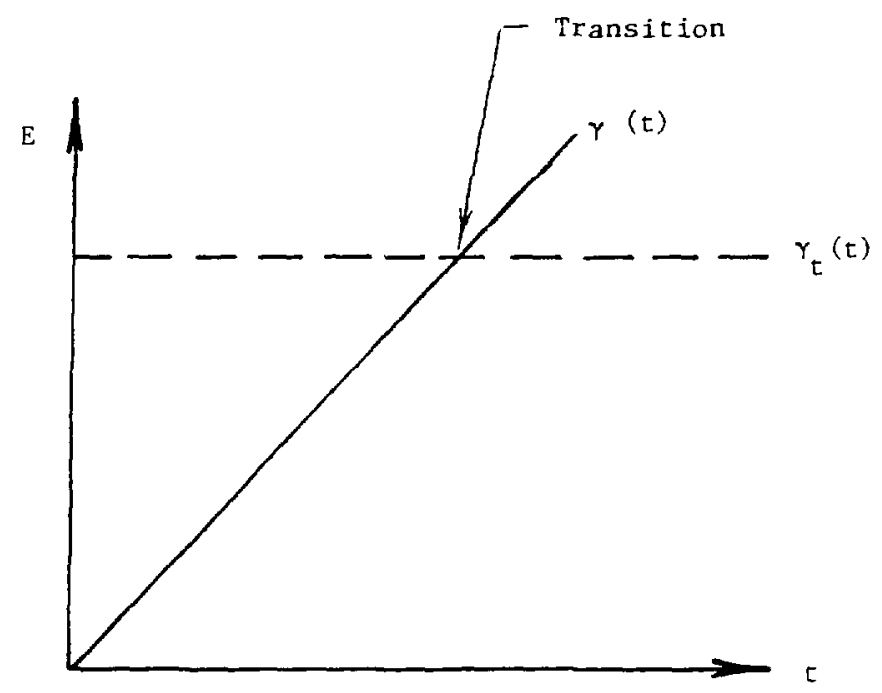

a) No $y_{t r}-$ jump

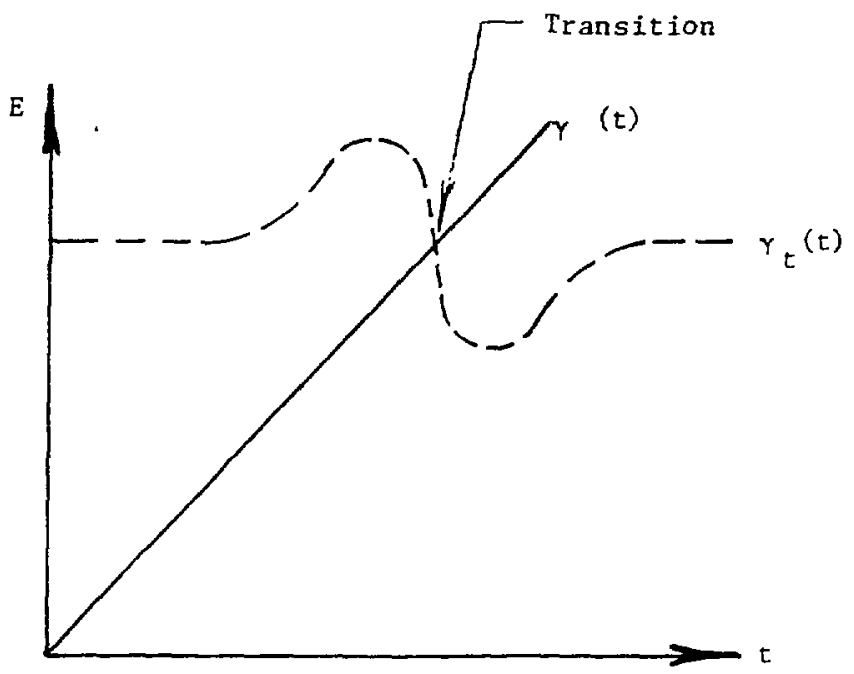

b) With $Y_{t r}-j u m p$

Fig. 25 Relattonship between particle energy

$\gamma$ and transition energy ${ }_{t r}$ 


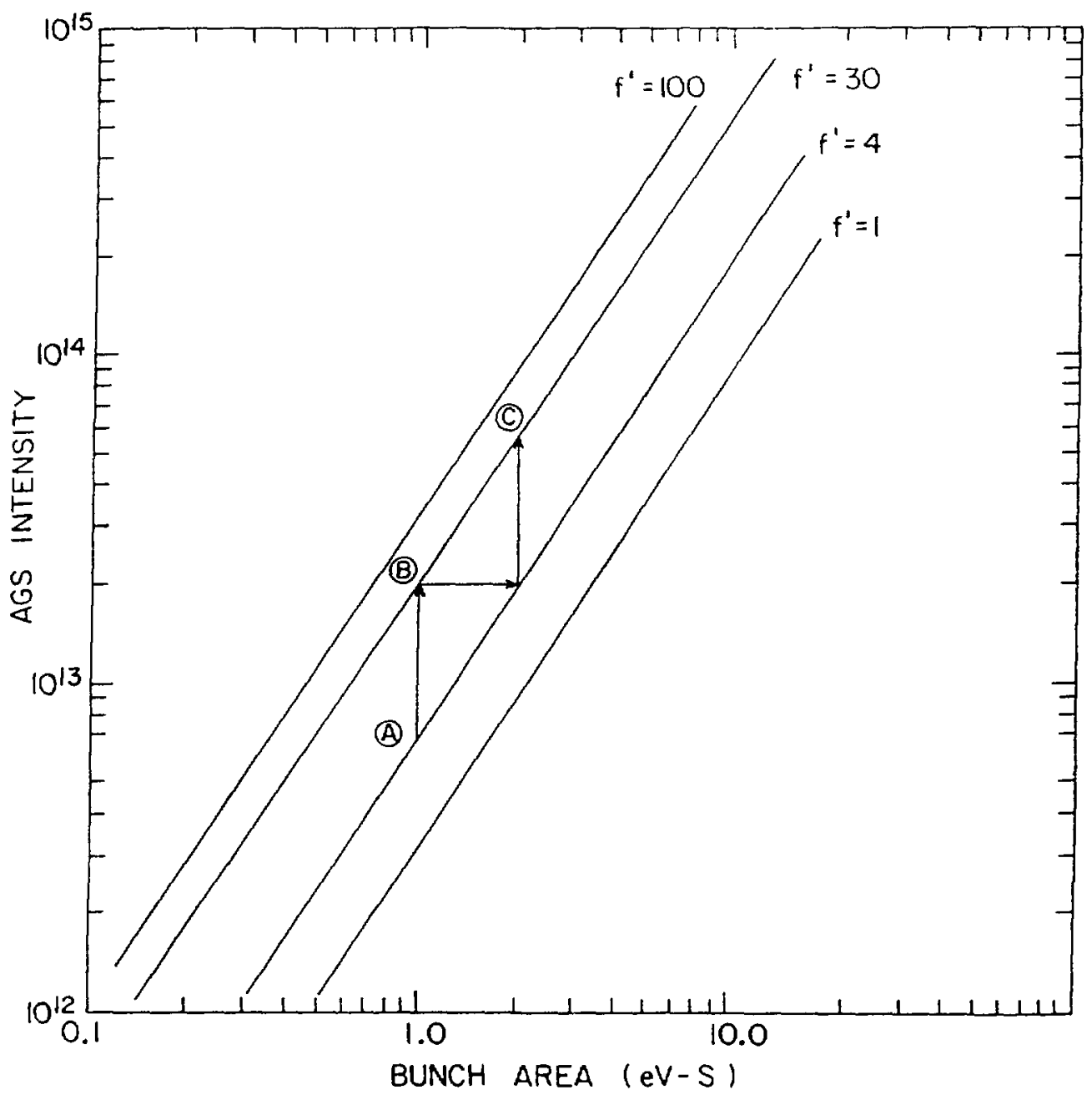

Fig. 26. Achievable AGS intensity as function of the strength of transition jump. 
bunch area, the tolerable intensity now is about $2 \times 10^{13}$. This should be compared with the empirical observation that, at $1.5 \times 10^{13}$, the AGS typically loses $5 \%$ of beam and suffers $50 \%$ blowup in passing through transition without $\gamma_{t r}$-jump.

With the addition of a booster, to run the AGS at $6 \times 10^{13} \mathrm{ppp}$, we need both a $\gamma_{t r}$-jump with $f^{\prime} \approx 30$ and controlled blowup to $2 \mathrm{eV}$-sec before transition.

\section{ACKNOWLEDGEMENT}

The author would like to thank F. Z. Khiari for help in generating the manuscript and for helpful discussions. 


\section{BIBLIOGRAPHY}

\section{1) INTRODUCTION}

1. V. Veksler, A New Method of Acceleration of Relativistic Particles, J. Phys. USSR 9: 153-9 (1945); also in The Development of A.G. Accelerators, M. S. Livingston, Ed., Dover, 1966.

2. E. M. MacMillan, The Synchrotron-A Proposed High Energy Particle Accelerator, Phys. Rev. 68: 143-4 (1945); also in Ibid.

3. F. T. Cole, Longitudinal Motion in Circular Accelerators, AIP Conf. Proc. 153, 44-83 (SLAC Summer School 1985).

2) DYNAMICS OF LONGITUDINAL MOTION

4. B. W. Montague, $R F$ Acceleration, CERN 77-13, 63-81.

5. K. H. Keich, Summary of Theory of Synchrotron Oscillations in A.G. Proton Synchrotrons, AADD-TN13, BNL, 1965.

6. F. T. Cole and L. P. Morton, Areas and Bunching Factors of Partially Filled Buckets, UCID, 10130, 1964.

7. J. Herrera, Particle Motion in the Alternating Gradient Synchrotron, Brookhaven Lectures on Accelerator Physics, Chap. 1, 1978.

\section{3) RF CAVITY FOR NONRELATIVISTIC SYNCHROTRONS}

8. F. G. Brockman, H. Van der Heide, and M. W. Louwerse, Ferroxcube for Proton Synchrotrons, Phillips Technical Review 20, 312-29 (1969).

9. J. Griffin, A Numerical Example of an RF Accelerating System, AIP Conf. Proc. 87, 564-582 (FNAL Summer School 1981).

10. M. Puglisi, Radio-Frequency Systems, These proceedings. 
11. G. Dome, RF Systems: Waveguides and Cavities, AIP Conf. Proc. 153, 1296-1412 (SLAC Summer School 1985).

4) LOW-LEVEL RF SYSTEM AND BEAM MANIPULATIONS

12. J. A. Maclachlan, Particle Tracking in $E--\phi$ Space as a Design Tool for Particle Accelerators, IEEE PAC Proc., March, 1987, 1007.

13. A. U. Luccio, F. Z. Khiari, and W. T. Weng, Simulation Studies of Longitudinal Beam Dynamics in the AGS Booster, EPAC, June $7-11,1988$, Rome, Italy.

14. K. Johnsen and C. Schmelzer, Beam Controlled Acceleration in Synchrotrons, in CERN Symposium on H. E. Accelerators, pp. 395-403, 1956.

15. D. Boussard, An Elementary Presentation of The PS Beam Control System, MPS/SR/Note 73-10.

16. E. Raka, Beam Acceleration and Control in The AGS, Brookhaven Lectures in Accelerator Physics, Chap. V, 1978.

17. K. W. Robinson, Stability of Beam in Radio-Frequency Systems, CEAL-1010, 1964

\section{5) SPACE-CHARGE EFFECTS}

18. I. Gumowski and K. H. Reich, Synchrotron Motion in the Presence of Space-Charge, CERN/SI/Int. DL/70-6.

19. A. Sorenssen, Crossing the Phase Transition in Strong-Focusing Proton Synchrotrons, Part. Accel. 6, 141-65 (1975).

20. A. Hoffman, Single-Beam Collective Phenomena-Longitudinal, CERN 77-13, 139-174. 
21. L. Ahrens et al., A $\gamma_{t r}$-Jump Scheme for the Brookhaven AGS, Acc. Div, TN-265, BNL, 1986. 